FERMILAB-TM-1720

\title{
Jet Energy Resolution of the SDC Detector
}

A. Para, A. Beretvas, K. Denisenko, N. Denisenko, D. Green and G. P. Yeh

Fermi National Accelerator Laboratory

P.O. Box 500

Batavia, Illinois 60510

W. Wu

Argonne National Laboratory

Argonne, Illinois 60439

H. Iso

University of Tsukuba

Ibaraki 305, Japan

December 6, 1990 


\section{Jet Energy Resolution of the SDC Detector}

SDC-90-00149(1990) Dec. 6, 1990

A. Para, A. Beretvas, K. Denisenko, N. Denisenko, D. Green, and G. P. Yeh

Fermi National Accelerator Laboratory, Batavia, Illinos, 65010

W. Wu

Argonne National Laboratory, Argonne, Mlinois, 60439

H. Iso

University of Tsukuba, Ibaraki 305, Japan

The SDC Simulation Group at Fermilab

\section{Abstract}

We have answered the PAC question ('Demonstrate the jet energy resolution of your proposed detector by studying decays $Z \rightarrow$ jet + jet and $Z^{\prime} \rightarrow$ jet + jet,$\left.M_{Z^{\prime}}=1 \mathrm{TeV} .^{\prime}\right)$ using a general program called SSCSIM. ${ }^{[1]}$ This program is a tool for investigating simple questions involving the relations between detector parameters and physics capabilities of a detector. A different package called ANLSIM developed by our colleagues at Argonne (see Appendix A) has also been used to answer this question. ${ }^{[2]}$ The results as expected are very similar. In this note we will try to document our procedures.

Our tentative conclusion from this study is that physics induced effects, out-of-cone fluctuations and underlying event fluctuations, dominate the resolution. Pushing the detector performance to the limits of technology improves the effective resolution by at most $20 \%$. 


\section{Physics Motivation}

Although inclusive jet physics is interesting in itself ${ }^{[3]}$, it is quite likely that the strongest demands concerning jet resolution will come from the study of multi-jet spectroscopy. At present much of CDF's efforts are involved in searching for a top quark decaying into electron plus jets, and in finding SUSY particles where the main signature is $\mathbb{E}_{\mathrm{T}}$ and jets. At the SSC energies W's and Z's are expected to be the most striking signatures of 'new physics'. The ability to tag events with the hadronic decay of the IVB's would greatly enhance the statistical power of our search for new, rare phenomena. In addition we will search for new, heavy objects like $Z^{\prime}$, decaying into two jets. Observation of the hadronic decay modes will be important in establishing the couplings of these new objects; for example exotic scenarios with heavy objects decoupled from the lepton sector.

\section{Process that have been Investigated using the package}

This package has been used to investigate many different physics issues. These include,'SDC at High Luminosity' ${ }^{[4]}$,'Dijet Spectroscopy at High Luminosity' [5], 'Muon Rates at the SSC'[6], and analysis of $\mathrm{t} \bar{t} \rightarrow \mathrm{b}$ jet jet $\bar{b}$ jet jet. [7]

The package has been run under both VMS and UNIX operating systems. There is also documentation for the package. ${ }^{[1]}$

\section{Event Generators}

We use ISAJET version $6.31^{[8]}$ to generate $\mathrm{Z}$ and $\mathrm{Z}^{\prime}$ via the Drell-Yan mechanism with an additional requirement that the jet axis be within $|\eta|<1$. The explicit input is given in TABLE I. We have studied the di-jet mass resolution at low $\mathrm{p}_{t}\left(50 \mathrm{GeV}<\mathrm{p}_{t}<60 \mathrm{GeV}\right)$ and at high $\mathrm{p}_{t}(500 \mathrm{GeV}<$ 
$\mathrm{p}_{t}<600 \mathrm{GeV}$ ) for both $\mathrm{Z}$ and $\mathrm{Z}^{\prime}$. For all 4 data sets we have produced files with high statistics (1000 events). The particles generated by the Monte Carlo program (ISAJET) are traced through the magnetic field to the inner surface of the calorimeter. The following detector effects are then simulated: transverse shower profile, energy resolution, non-linearity of the response of the hadron calorimeter, cracks in the detector,etc. The output of the program is the energy deposited in the calorimeter. The process is repeated for all events while histograms are filled using HBOOK. ${ }^{[9]}$ At Argonne the same process has been simulated using PYTHIA version 4.9. ${ }^{[10]}$

\section{HEP format and Simulation code}

The output from ISAJET is written in HEP format (this is the particle numbering scheme proposed

by the Particle Data Group). ${ }^{[1]}$ The files are then analyzed by our simulation code. The Simulation code is all written in standard fortran (F77) and is maintained using Patchy.

\section{Jets from $\mathrm{Z}$}

We get an intuitive understanding of jets from a transverse energy lego plot (see Figure 1a-h). The first two events are plotted for each of the four cases ( $Z$ low $\mathrm{p}_{t}, \mathrm{Z}$ high $\mathrm{p}_{t}, \mathrm{Z}^{\prime}$ low $\mathrm{p}_{t}$, and $\mathrm{Z}^{\prime}$ high $\mathrm{p}_{t}$ ) considered. At low $\mathrm{p}_{t}$, most of the time, it is easy to locate the two jets (see Fig. 1a). However there are times when it is difficult to see the second jet because it does not contain enough transverse energy (see Fig. 1b). Other events, not shown, exist when there are jets other than those associated with the $\mathrm{Z}$. At high $\mathrm{p}_{t}$ we see a strong pencil-like collimation of the jets, due to the Lorentz boost. Fig. 1c shows a case when the two jets are easily distinguished, the picture also shows two other jets. In the next lego plot ( Fig. 1d) the two jets have merged. This is almost 
always the case for a $Z$ at high $\mathrm{p}_{t}$. A lego plot for a $Z^{\prime}$ at low $\mathrm{p}_{t}$ is given in Fig. le. Again we notice a large jet not associated with the $Z^{\prime}$. Fig. If is a nice clean event. Two typical cases for a $Z^{\prime}$ at high $p_{t}$ are given in figures $1 g$ and $1 \mathrm{~h}$. We see that in all cases except the $Z$ at low $p_{t}$ the jets show pencil-like collimation. The size of the jet in eta phi space, $R=\sqrt{\delta \eta^{2}+\delta \phi^{2}}$, for the $\mathrm{Z}$ is indicated by the distribution $\frac{d E}{d R}$ given in Figure $2 \mathrm{a}(\mathrm{b})$ for low (high) $\mathrm{p}_{t}$. The corresponding plots for the $Z^{\prime}$ are shown in Figures 2c and 2d.

\section{Jet Resolution}

In this section we study the energy and angular resolution for hadronic jets, as well as the di-jet mass resolution. Jets are defined as local clusters of energy in the calorimeter. We cluster energy in a cone of a given radius in eta phi space. The cone is centered on the direction of the primary quark as taken from the event generator information. It is expected that for high $\mathrm{p}_{t}$ jets this is reasonably close to the results of the clustering algorithm we will eventually use. ${ }^{[12]}$ It is also an underestimate of the irreducible errors due to physics processes and, therefore, will accentuate any detector dependent effects.

The jet energy and angular resolution depends strongly on the jet transverse momentum. Di-jet mass reconstruction and resolution will depend on the Lorentz boost of the parent object. At low $\mathrm{p}_{t}$ we form the invariant mass of two jets, whereas for highly boosted parents the invariant mass of the coalesced jet provides the best estimate of the parent mass.

The jet 4-momentum vector was calculated by summing all calorimetric cells within the cone (with a typical radius of 0.7 ), treating all cells as massless particles.[13] The invariant mass of the two jet system was calculated as $M_{j j}=\left[\left(E_{1}+E_{2}\right)^{2}-\left(P_{1}+P_{2}\right)\right]^{1 / 2}$. This two jet method of 
calculating the mass is used for the $Z$ at low $p_{t}$ and the $Z^{\prime}$ at both low and high $p_{t}$. For the $Z$ at high $\mathbf{p}_{t}$, we calculated a single jet mass as $\mathrm{M}_{\mathrm{jj}}=\left[\left(\mathrm{E}_{1}\right)^{2}-\left(\mathbf{P}_{1}\right)^{2}\right]^{1 / 2}$, using a cone centered on the Z laboratory direction.

\section{Detector Simulation}

In our analysis we consider simplified model of the detector. ${ }^{[1]}$ The calorimeter is assumed to have an inner radius of 2.3 meters and a total length of 9 meters. ${ }^{[14]}$ The granularity of the calorimeter is taken as .05 in both $\phi$ and $\eta$. Hadron showers are spread transversely using the parameterization of ref [15]. Electromagnetic showers are taken to have a typical width of $1 \mathrm{~cm}$. The hadron energy resolution is normally assumed to be $50 \% / \sqrt{\mathrm{E}} \oplus 4 \%$, and the electromagnetic energy resolution is taken as $20 \% / \sqrt{\mathrm{E}} \oplus 1 \%$. We note that the jet energy resolution is dominated by the resolution of the hadron component. The constant term in the resolution was added in quadrature to the stochastic one, and the energy of every particle was smeared with the resulting resolution. The magnetic field was assumed to be purely axial, with a strength of 1.8 Tesla. The non linear response of the hadron calorimeter due to a possible imperfect compensation was simulated using an ansatz of D. Groom. [16]

\section{Detector Independent Results}

The measurement accuracy of the jet parameters has several components; some of them are detectordependent, others are imposed by the physics. Before optimizing the detector-dependent ingredients of the resolution function it is important to understand the physics imposed limitations.

Measurement of hadronic jets consists of the measurement of the jet energy and its direction. 
The Di-jet mass resolution results from a combination of both the energy and angular resolution, with a weight which depends on the Lorentz boost of the system. The angular resolution becomes important at very large $\mathrm{p}_{t}$.

Most of the mass distributions and resolution functions are non-gaussian. They typically have long, asymmetric tails. We have decided to use as a figure of merit the sigma based on a Gaussian fit in a region that contains $75 \%$ of the events, centered on the peak channel. The resolution of the jet direction measurement is characterized by an angle $\alpha$, such that $66 \%$ of the jets have their axis reconstructed with an error smaller than $\alpha$. Figure 3a-d gives the error on the jet direction. Figures 4a-d gives the angle between the jet and it's daughters. Figure $5 \mathrm{a}-\mathrm{d}$ is a plot of $\frac{E_{\text {jet }}-E_{\text {(reconstucted })_{j e t}}}{E_{j e t}}$ which indicates the jet energy resolution. Figure 6a-d shows typical reconstructed mass distributions for $\mathrm{Z} \rightarrow$ jet + jet. In Table $\Pi$ we present results on the di-jet mass resolution and on the jet energy resolution as a function of detector parameters for both $\mathrm{Z}$ and $\mathrm{Z}^{\prime}$ at both high and low $\mathrm{p}_{T}$. The high pr $\mathrm{Z}$ case is treated in more detail, as this is the most likely example of a possible use for physics analysis. Our results demonstrate that the dominant contribution to the energy and/or mass resolution for these process comes from the physics.

\subsection{Cone size and underlying events}

Jets are defined as cones in eta-phi space. In the jet fragmentation process there will be particles emitted outside the cone. Fluctuations in this component will induce energy and angular resolution effects. This effect can be reduced by increasing the cone size, but this leads, inturn, to increased fluctuations of the contribution of the underlying event and of the contribution of other events from the same bunch crossing. The latter effect will become especially serious at high luminosities. Resolution must be optimized for particular experimental conditions, taking into account integration 
time, selecting suitable energy thresholds, selecting cone sizes etc...; for the time being we ignore these problems and simply use a cone of radius 0.7 . Table III gives the resolution for the $\mathrm{Z}^{\prime}$ for cone sizes of $0.7,0.5$ and 0.3 . It is interesting to observe that a cone size of 0.7 gives significantly better resolution than a cone size of 0.5 for both low and high $\mathrm{p}_{t}$.

\subsection{Fluctuations due to Neutrinos}

Another contribution to the detector-independent resolution is due to fluctuations in the energy fraction carried away by undetected neutrinos from semileptonic decays of heavy quarks inside the jet. Neutrinos will produce a long tail of undermeasured jet energies, or low invariant masses of the di-jet system. ${ }^{[17]}$ They will therefore reduce the efficiency of the tagging procedure by some $20 \%$, but they will not contribute to the 'almost gaussian' part of the resolution function.

\section{Detector induced resolution effects}

In the following we examine several detector-dependent contributions. In order to verify that the proposed performance of the SDC calorimeter will not significantly degrade the di-jet mass resolution.

\subsection{Detector Granularity}

The detector granularity, within wide limits $(0.03$ to 0.10$)$, has relatively little effect on the mass

resolution. At high $\mathrm{p}_{t}$ one starts to see an effect with a cell size of 0.10 . From other studies [7],[18] we know that there is a large effect for cell sizes greater than 0.10 . Boosted jets have a mass which is more sensitive to angular errors, than jets at low $\mathrm{p}_{t}$. 


\subsection{Detector Thresholds}

Another contribution to the resolutions will be due to the detector threshold. We have investigated this issue and the results are given in Table IV. ${ }^{[19]}$ We see that for low $p_{t}$ resolution is degraded when the threshold exceeds $500 \mathrm{MeV}$. At high $P_{t}$ the threshold does not effect the mass resolution.

\subsection{Magnetic Field}

At low $p_{t}$ the fluctuations in the jet fragmentation dominate and the effect of the magnetic field is small. At high $P_{t}$ the magnetic field effects are noticeable and the mass resolution increases from $5.0 \%$ to $6.4 \%$. However, by using tracking information almost all the mass resolution can be recovered. $[20]$

\subsection{Intrinsic energy resolution (constant + stochastic)}

Note that the EM resolution is always very good on the scale discussed here, and only the hadronic resolution is relevant. Table $\mathrm{V}$ gives the $\mathrm{Z}$ mass resolution at low $\mathrm{p}_{t}$ for three different choices of the hadronic energy resolution. For energy resolution in the range $30 \%-70 \%$ the mass resolution changes very little, on the other hand, a resolution of $150 \% / \sqrt{\mathrm{E}} \oplus 7.5 \%$ degrades the mass resolution substantially.

\subsection{Non-linearity of the hadron calorimeter}

The non-linearity of the hadron calorimeter, induced by $\mathrm{e} / \mathrm{h}=1.3$, does not substantially change the di-jet mass resolution ( see Table II). If the detector consists of regions with different $\mathrm{e} / \mathrm{h}$ one must be careful in combining the data from the different regions because the mass is shifted. It is most likely that one needs to make fiducial cuts to avoid the regions near the boundaries. 


\section{Conclusions}

In case of lower $p_{t}$ Z's the mass resolution is dominated by the fluctuation of energy outside the 0.7 cone and fluctuations of the underlying event. A perfect detector could measure the $Z$ mass with a resolution of $8 \%$. Detector induced effect worsen this resolution to $8.5-9 \%$. Even a substantial non-linearity of the hadron calorimeter, induced by $\mathrm{e} / \mathrm{h}=1.3$, does not change this conclusion. ${ }^{[18]}$ In case of high pr Z's, all particles are well contained in the cone. However, fluctuations of the underlying event bring the resolution from $1.7 \%$ up to $4.3 \%$. Detector induced effects increase the resolution to $6-7 \%$, although the resolution depends very weakly on detector performance within the studied limits. There is not much to be gained by pushing detector performance to the limits of technology, at least in the cases we have studied. High mass objects are measured substantially better, owing to the high $\mathbf{p}_{T}$ of the jets in question and the large opening angle. A typical mass resolution is of order $3-5 \%$, but it is again very weakly dependent on the detector

\section{Appendix A}

Table A gives both our results and those of the Argonne group for $\mathrm{Z} \rightarrow$ jet + jet at both low and high $\mathbf{p}_{t}$. The Argonne algorithm, the second method, uses all clusters that have their centroid within the clustering radius from the original quark direction (only the $75 \%$ of the events around the peak are used). They also use a seed tower of $2.0 \mathrm{GeV}$ and require a threshold of $0.1 \mathrm{GeV}$. We notice that the agreement at low $p_{t}$ is excellent and that at high $p_{t}$ is quit good. We quota a sigma for our answer because we know the generated mass for each event. Our sigma is a measure of the response of the detector and does not depend on the width of the $\mathrm{Z}$ given in the Monte Carlo. 


\section{References}

[1] A. Beretvas et al., SSCSIM user guide (internal note SSC-SDC-Fermilab-31).

[2] R. Blair et al., (internal notes SSC-SDC-Fermilab-24, 30, 42, and 50).

[3] F. Abe et al. Phys. Rev. Lett. 62, 616 (1989), Phys. Rev. Lett. 62, 3020 (1989); Phys. Rev. Lett. 64, 157 (1990) and Phys. Rev. Lett. 64, 348 (1990).

[4] D. Green (Fermilab-Pub-90/110, May 1990).

[5] D. Green (Fermilab-Conf-90/151, July 1990).

[6] D. Green (Fermilab-Pub-90/18, Jan 1990).

[7] A. Para (work in progress).

[8] F. Page and S. D. Protopopescu, ISAJET Monte Carlo, BNL report No. BNL 38034, 1986 (unpublished).

[9] R. Brun and D. Lienart, HBOOK User Guide, Cern Program Library Entry Y250 (Oct. 1987) R. Brun et al., PAW-Physics Analysis Workstation, Cern Program Library Entry Q121 (Oct. 1989).

[10] H. U. Bengtsson, and T. Sjöstrand, 'Hadron-hadron high-p $\mathrm{p}_{\perp}$ scattering'.

[11] H. Iso, HEP list of Particles (internal note SSC-SDC-Fermilab-23).

[12] The Argonne code uses a standard Jet clustering algorithm.

[13] The energy is summed over all the cells inside the cone $\mathrm{E}=\sum \mathrm{E}_{\boldsymbol{i}}, \mathrm{p}_{\boldsymbol{z}}=\sum \mathrm{E}_{\boldsymbol{i}} \cos \left(\theta_{i}\right), \ldots$, where $\theta=2 \tan ^{-1}\left(\mathrm{e}^{-\eta}\right)$. 
[14] Solenoid Detector Collaboration, EOI, p11, May 1990.

[15] F. Binon et al. NIM 206 (1983), 373.

[16] D. Groom, Proceedings of the Workshop on Calorimetry for the Superconducting Super Collider, Tuscaloosa, Alabama, 13-17 March 1989, ed. R. Donaldson and M. G. D. Gilchriese, World Scientific (June 1990), 59-75.

[17] N. Denisenko and K. Denisenko (internal note SSC-SDC-Fermilab-47).

[18] W. Wu (internal note SSC-SDC-Fermilab-32).

[19] W. Wu (internal note SSC-SDC-Fermilab-52).

[20] W. Wu (internal note SSC-SDC-Fermilab-53). 


\section{Figure Captions}

Figure 1(a-h)-Lego Plots The axes of the grid represent the azimuthal angle around the beam line, $\phi$, and the pseudorapidity $\eta$. The height of each cell is proportional to its $\mathrm{E}_{t}$.

1(a-b) $\mathrm{Z}$ at low $\mathrm{p}_{t}$, (c-d) $\mathrm{Z}$ at high $\mathrm{p}_{t}$, (e-f) $\mathrm{Z}^{\prime}$ at low $\mathrm{p}_{t},(\mathrm{~g}-\mathrm{h}) \mathrm{Z}^{\prime}$ at high $\mathrm{p}_{t}$.

Figure 2(a-d) Plot of $d E / d R$ versus $R$ where $R=\sqrt{\delta \eta^{2}+\delta \phi^{2}}$.

(a) $\mathrm{Z}$ at low $\mathrm{p}_{t}$, (b) $\mathrm{Z}$ at high $\mathrm{p}_{t}$, (c) $\mathrm{Z}^{\prime}$ at low $\mathrm{p}_{t}$, (d) $\mathrm{Z}^{\prime}$ at high $\mathrm{p}_{t}$.

Figure 3(a-d) Distribution of the error on the jet direction.

(a) $\mathrm{Z}$ at low $\mathrm{p}_{t}$, (b) $\mathrm{Z}$ at high $\mathrm{p}_{t}$, (c) $\mathrm{Z}^{\prime}$ at low $\mathrm{p}_{t}$, (d) $\mathrm{Z}^{\prime}$ at high $\mathrm{p}_{t}$.

Figure 4(a-d) Distribution of the angle (radians) between the jet and its daughters.

(a) $\mathrm{Z}$ at low $\mathrm{p}_{t}$, (b) $\mathrm{Z}$ at high $\mathrm{p}_{t}$, (c) $\mathrm{Z}^{\prime}$ at low $\mathrm{p}_{t}$, (d) $\mathrm{Z}^{\prime}$ at high $\mathrm{p}_{t}$.

Figure $5(\mathrm{a}-\mathrm{d})$ Distribution for $\frac{E_{\text {jet }}-E(\text { reconstructed })_{\text {jet }}}{E_{\text {jet }}}$.

(a) $\mathrm{Z}$ at low $\mathrm{p}_{t}$, (b) $\mathrm{Z}$ at high $\mathrm{p}_{t}$, (c) $\mathrm{Z}^{\prime}$ at low $\mathrm{p}_{t}$, (d) $\mathrm{Z}^{\prime}$ at high $\mathrm{p}_{t}$.

Figure 6(a-d) Distribution of jet-jet reconstructed mass.

(a) $\mathrm{Z}$ at low $\mathrm{p}_{t}$, (b) $\mathrm{Z}$ at high $\mathrm{p}_{t}$, (c) $\mathrm{Z}^{\prime}$ at low $\mathrm{p}_{t}$, (d) $\mathrm{Z}^{\prime}$ at high $\mathrm{p}_{t}$. 
TABLE I

\begin{tabular}{|c|c|}
\hline \multicolumn{2}{|c|}{ ISAJET input for the Drell-Yan Process } \\
\hline $\mathrm{Z}^{0}$ at low $\mathrm{p}_{t}$ & $\mathrm{Z}^{0 \prime}$ at low $\mathrm{p}_{t}$ \\
\hline \multicolumn{2}{|c|}{$\mathrm{pp} \rightarrow \mathrm{Z} \rightarrow$ jet + jet } \\
\hline \multicolumn{2}{|c|}{$40000 ., 1000,0,0 /$} \\
\hline \multicolumn{2}{|c|}{ DRELLYAN } \\
\hline \multicolumn{2}{|l|}{ BEAMS } \\
\hline \multicolumn{2}{|l|}{ 'P','P'/ } \\
\hline \multicolumn{2}{|l|}{ QMW } \\
\hline $80 ., 100 . /$ & $900 ., 1100 . /$ \\
\hline \multicolumn{2}{|l|}{ YW } \\
\hline \multicolumn{2}{|l|}{$-1 ., 1 .,-1 ., 1 . /$} \\
\hline \multicolumn{2}{|l|}{ QTW } \\
\hline \multicolumn{2}{|l|}{$50 ., 60 . /$} \\
\hline \multicolumn{2}{|l|}{ WMASS } \\
\hline $80 ., 91.2 /$ & $887.4,1000 . /$ \\
\hline \multicolumn{2}{|l|}{ WTYPE } \\
\hline \multicolumn{2}{|l|}{ 'Zo'/ } \\
\hline \multicolumn{2}{|l|}{ JETTYPE1 } \\
\hline \multicolumn{2}{|l|}{ 'QUARKS' } \\
\hline \multicolumn{2}{|l|}{ JETTYPE2 } \\
\hline \multicolumn{2}{|l|}{ 'QUARKS' } \\
\hline \multicolumn{2}{|l|}{ NTRIES } \\
\hline \multicolumn{2}{|l|}{$5000 /$} \\
\hline \multicolumn{2}{|l|}{ END } \\
\hline STOP & \\
\hline
\end{tabular}


TABLE II.

\begin{tabular}{|c|c|c|c|c|}
\hline \multicolumn{5}{|l|}{ Di-jet mass resolution and jet energy resolution } \\
\hline case & $\mathrm{m}(\operatorname{Rec}) / \mathrm{m}(\mathrm{Gen})$ & $\operatorname{sigma}(m) / m$ & $\Delta \mathrm{E} / \mathrm{E}$ & $\operatorname{sigma}(E) / E$ \\
\hline \multicolumn{5}{|l|}{$\mathrm{Z}^{0}$ at low $\mathrm{p}_{t}$} \\
\hline default & 0.928 & 0.088 & 0.045 & 0.091 \\
\hline cone 0.9 & 1.011 & 0.088 & -0.007 & 0.094 \\
\hline cone 1.1 & 1.078 & 0.121 & -0.064 & 0.105 \\
\hline cell 0.03 & 0.927 & 0.087 & 0.049 & 0.092 \\
\hline cell 0.10 & 0.924 & 0.093 & 0.051 & 0.089 \\
\hline resolution $30 \% / \sqrt{E} \oplus 2 \%$ & 0.927 & 0.083 & 0.046 & 0.077 \\
\hline $\mathrm{e} / \mathrm{h} 1.3$ & 0.809 & 0.083 & 0.170 & 0.085 \\
\hline no $B$ field & 0.984 & 0.095 & -0.010 & 0.097 \\
\hline perfect detector, cell $0.01, Z^{0}$ only & 0.911 & 0.053 & 0.067 & 0.050 \\
\hline perfect detector, cell $0.01, Z^{0}$ only, cone 0.9 & 0.944 & 0.043 & 0.036 & 0.037 \\
\hline perfect detector, cell 0.01 & 0.994 & 0.079 & -0.015 & 0.063 \\
\hline perfect detector, cell 0.01 , cone 0.9 & 1.078 & 0.086 & -0.072 & 0.071 \\
\hline \multicolumn{5}{|l|}{$\mathrm{Z}^{0}$ at high $\mathrm{p}_{t}$} \\
\hline default & 1.069 & 0.064 & -0.002 & 0.024 \\
\hline cell 0.03 & 1.063 & 0.064 & 0.000 & 0.024 \\
\hline cell 0.10 & 1.088 & 0.071 & -0.002 & 0.024 \\
\hline resolution $30 \% / \sqrt{\mathrm{E}} \oplus 2 \%$ & 1.073 & 0.059 & -0.002 & 0.017 \\
\hline $\mathrm{e} / \mathrm{h} 1.3$ & 0.949 & 0.058 & 0.104 & 0.030 \\
\hline no B field & 1.051 & 0.050 & -0.011 & 0.023 \\
\hline perfect detector, cell $0.01, Z^{0}$ only & 0.972 & 0.017 & 0.008 & 0.010 \\
\hline perfect detector, cell 0.01 & 1.022 & 0.043 & -0.011 & 0.010 \\
\hline \multicolumn{5}{|l|}{$\mathrm{Z}^{0 \prime}$ at low $\mathrm{p}_{t}$} \\
\hline default & 0.970 & 0.032 & 0.024 & 0.032 \\
\hline cell 0.10 & 0.969 & 0.033 & 0.024 & 0.032 \\
\hline resolution $30 \% / \sqrt{E} \oplus 2 \%$ & 0.970 & 0.030 & 0.024 & 0.025 \\
\hline $\mathrm{e} / \mathrm{h} 1.3$ & 0.869 & 0.036 & 0.128 & 0.041 \\
\hline no B field & 0.977 & 0.036 & 0.014 & 0.032 \\
\hline \multicolumn{5}{|l|}{$\mathrm{Z}^{0 \prime}$ at high $\mathrm{p}_{t}$} \\
\hline default & 0.972 & 0.049 & 0.014 & 0.037 \\
\hline cell 0.03 & 0.968 & 0.054 & 0.016 & 0.038 \\
\hline cell 0.10 & 0.974 & 0.048 & 0.015 & 0.037 \\
\hline resolution $30 \% / \sqrt{\mathrm{E}} \oplus 2 \%$ & 0.973 & 0.049 & 0.014 & 0.030 \\
\hline $\mathrm{e} / \mathrm{h} 1.3$ & 0.874 & 0.046 & 0.116 & 0.042 \\
\hline no B field & 0.984 & 0.045 & 0.005 & 0.032 \\
\hline
\end{tabular}


TABLE III.

\begin{tabular}{lcccc}
\hline \hline Di-jet mass resolution & for different cone sizes & & & \\
\hline & & & & \\
case & $\mathrm{m}(\mathrm{Rec}) / \mathrm{m}(\mathrm{Gen})$ & $\mathrm{sigma}(\mathrm{m}) / \mathrm{m}$ & $\Delta \mathrm{E} / \mathrm{E}$ & $\operatorname{sigma}(\mathrm{E}) / \mathrm{E}$ \\
\hline $\mathrm{Z}^{0}$ at low $\mathrm{p}_{t}$ & & & & \\
\hline & & & & \\
default, cone 0.7 & 0.928 & 0.088 & 0.045 & 0.091 \\
cone 0.9 & 1.011 & 0.088 & -0.007 & 0.094 \\
cone 1.1 & 1.078 & 0.121 & -0.064 & 0.105 \\
\hline $\mathrm{Z}^{0}$ at high $\mathrm{p}_{t}$ & & & & \\
\hline & & & & \\
default, cone 0.7 & 1.069 & 0.064 & -0.002 & 0.024 \\
cone 0.9 & 1.132 & 0.084 & -0.009 & 0.029 \\
cone 1.1 & 1.213 & 0.119 & -0.016 & 0.030 \\
\hline $\mathrm{Z}^{0 \prime}$ at low $\mathrm{p}_{t}$ & & & & \\
\hline & & & & \\
cone 0.3 & 0.882 & 0.073 & 0.056 & 0.040 \\
cone 0.5 & 0.942 & 0.051 & 0.035 & 0.033 \\
default, cone 0.7 & 0.970 & 0.032 & 0.024 & 0.032 \\
\hline $\mathrm{Z}^{0 \prime}$ at high $\mathrm{p}_{t}$ & & & & \\
\hline & & & & \\
cone 0.3 & 0.874 & 0.077 & 0.071 & 0.072 \\
cone 0.5 & 0.935 & 0.056 & 0.039 & 0.041 \\
default, cone 0.7 & 0.972 & 0.049 & 0.014 & 0.037 \\
\hline \hline
\end{tabular}


TABLE IV.

\begin{tabular}{lcc}
\hline \hline Di-jet mass resolution as a function of minimum cell energy & \\
\hline & & \\
case & $\mathrm{m}(\mathrm{Rec}) / \mathrm{m}(\mathrm{Gen})$ & sigma(m)/m \\
\hline $\mathrm{Z}^{0}$ at low $\mathrm{p}_{t}$ & & \\
\hline & & \\
Minimum Cell Energy (GeV) & 0.92 & 0.092 \\
default 0.0 & 0.87 & 0.098 \\
0.1 & 0.83 & 0.092 \\
0.2 & 0.72 & 0.112 \\
0.5 & 0.59 & 0.129 \\
1.0 & & \\
\hline $\mathrm{Z}^{0}$ at high $\mathrm{p}_{t}$ & & \\
& & \\
Minimum Cell Energy (GeV) & & \\
default 0.0 & 1.07 & 0.068 \\
0.1 & 1.05 & 0.067 \\
0.2 & 1.05 & 0.062 \\
0.5 & 0.99 & 0.067 \\
1.0 & 0.95 & 0.069 \\
\hline \hline
\end{tabular}


TABLE V.

\begin{tabular}{lcccc}
\hline \hline Di-jet mass resolution & for different & hadron energy & resolutions & \\
\hline \hline & & & & \\
case & $\mathrm{m}(\mathrm{Rec}) / \mathrm{m}(\mathrm{Gen})$ & sigma $(\mathrm{m}) / \mathrm{m}$ & $\Delta \mathrm{E} / \mathrm{E}$ & sigma(E)/E \\
\hline $\mathrm{Z}^{0}$ at high $\mathrm{p}_{t}$ & & & & \\
\hline & & & & \\
$\mathbf{3 0 \%} / \sqrt{\mathrm{E}} \oplus 2 \%$ & 1.073 & 0.059 & -0.002 & 0.017 \\
$50 \% / \sqrt{\mathrm{E}} \oplus 3 \%$, default & 1.069 & 0.064 & -0.002 & 0.024 \\
$70 \% / \sqrt{\mathrm{E}} \oplus 4 \%$ & 1.065 & 0.072 & 0.000 & 0.031 \\
$150 \% / \sqrt{\mathrm{E}} \oplus 7.5 \%$ & 1.063 & 0.102 & -0.001 & 0.066 \\
\hline \hline
\end{tabular}


TABLE A.

\begin{tabular}{lccc}
\hline \hline Comparison (Fermilab/Argonne) & \multicolumn{3}{c}{ Di-jet width and mass } \\
\hline \hline & sigma $(\mathrm{m}) / \mathrm{m}$ & width $(\mathrm{GeV})$ & Mass $(\mathrm{Rec})(\mathrm{GeV})$ \\
\hline case & & & \\
\hline $\mathrm{Z}^{0}$ at low $\mathrm{p}_{t}$ & & & \\
\hline & 0.088 & 8.44 & 84.2 \\
default & 0.093 & 9.06 & 80.1 \\
Threshold $0.1(\mathrm{GeV})$ & $\mathrm{x}$ & 9.1 & 80.5 \\
Argonne & & & \\
\hline $\mathrm{Z}^{0}$ at high $\mathrm{p}_{t}$ & & & 98.0 \\
& 0.064 & 6.24 & 96.5 \\
default & 0.064 & 6.19 & 89.4 \\
Threshold $0.1(\mathrm{GeV})$ & $\mathrm{x}$ & 6.9 & \\
Argonne & & & \\
\hline \hline
\end{tabular}




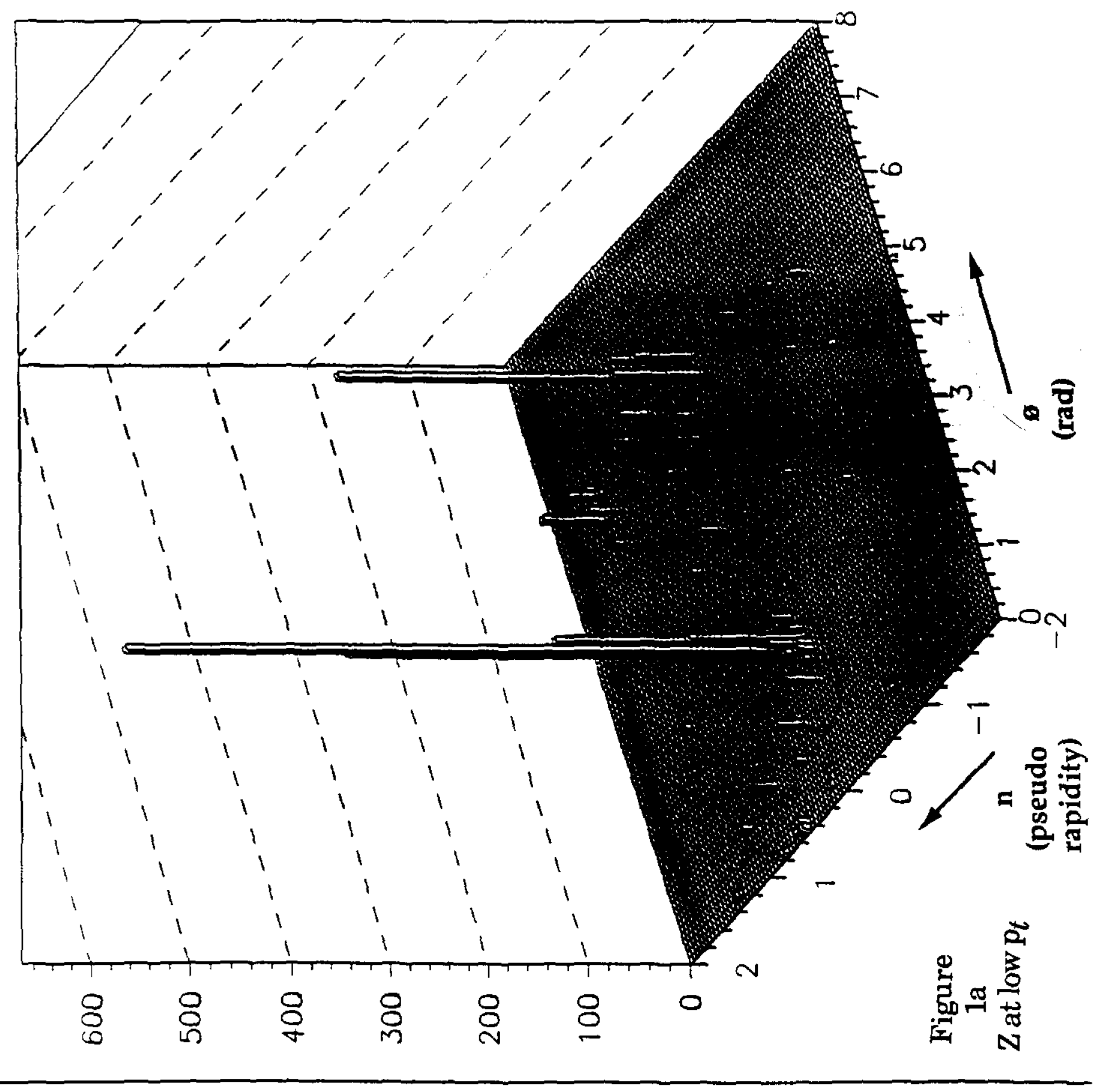




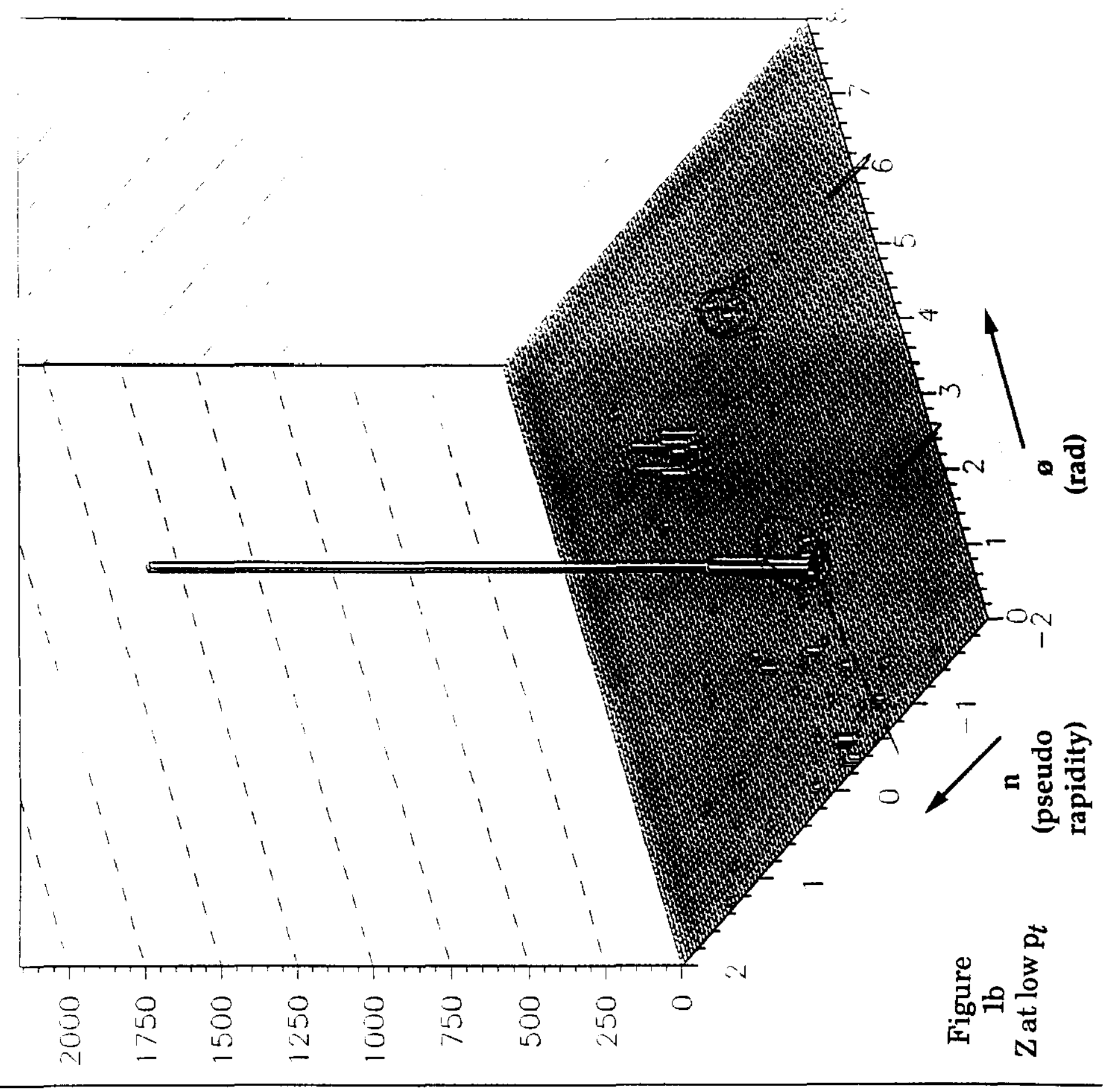




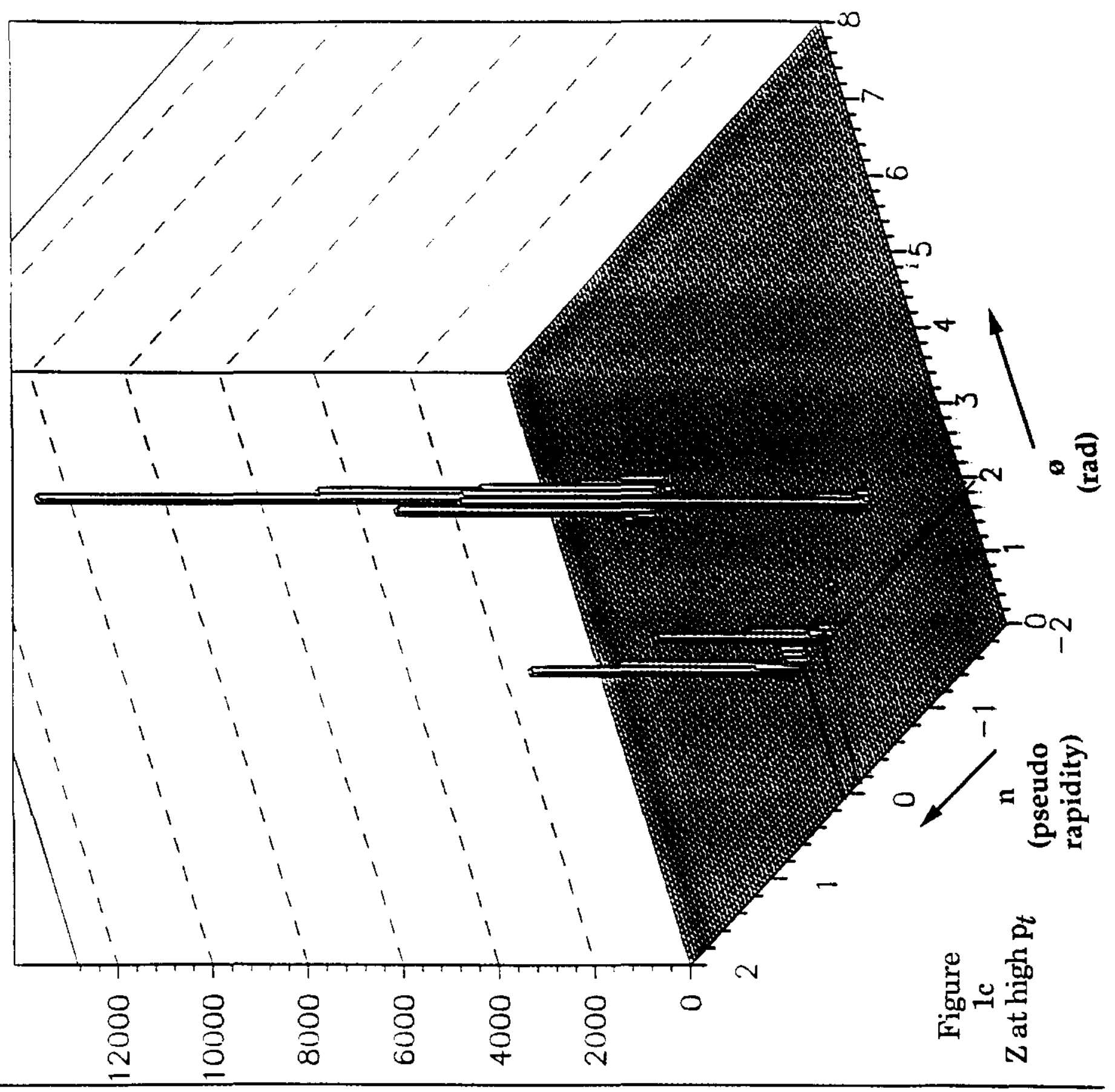




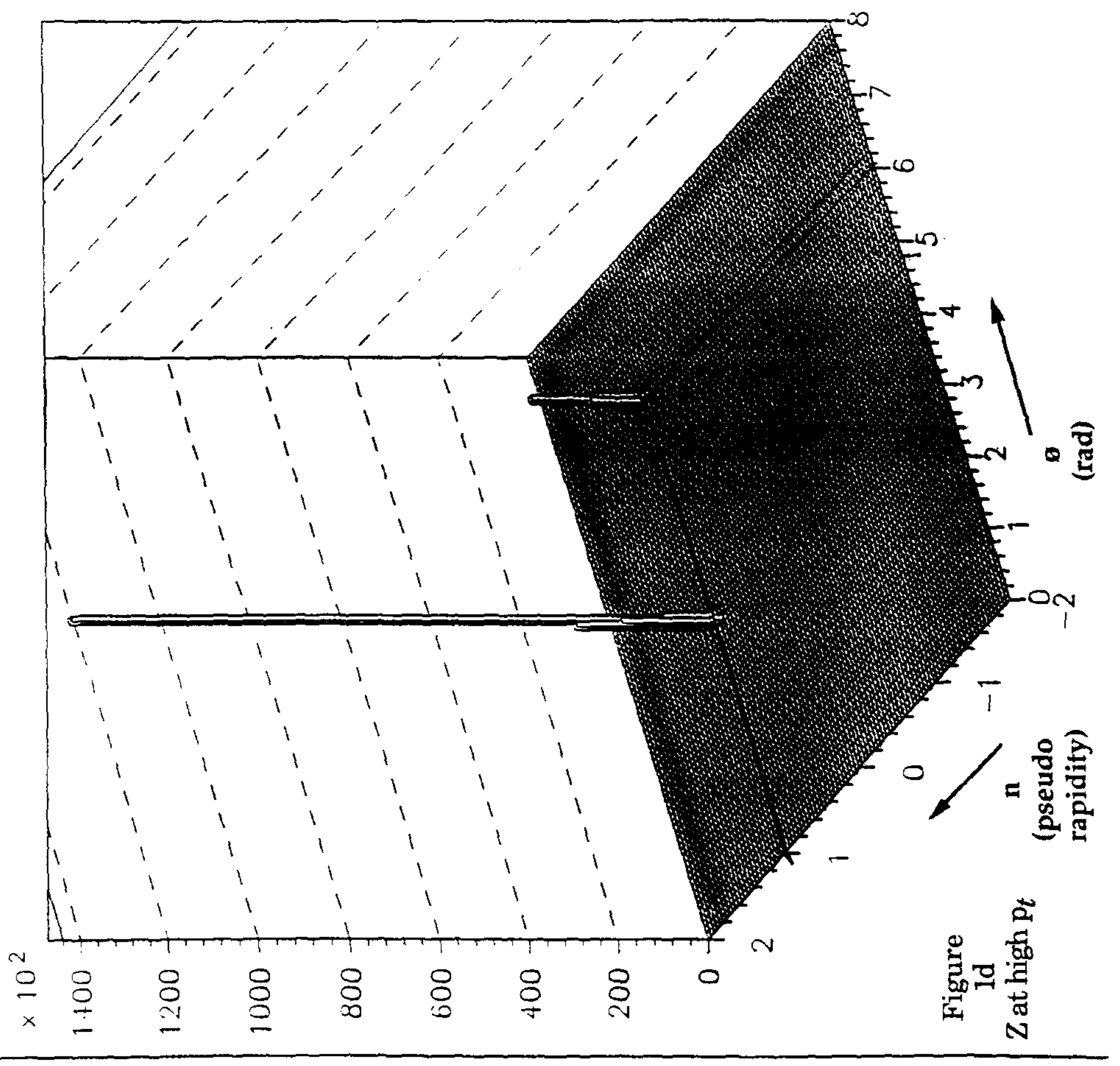




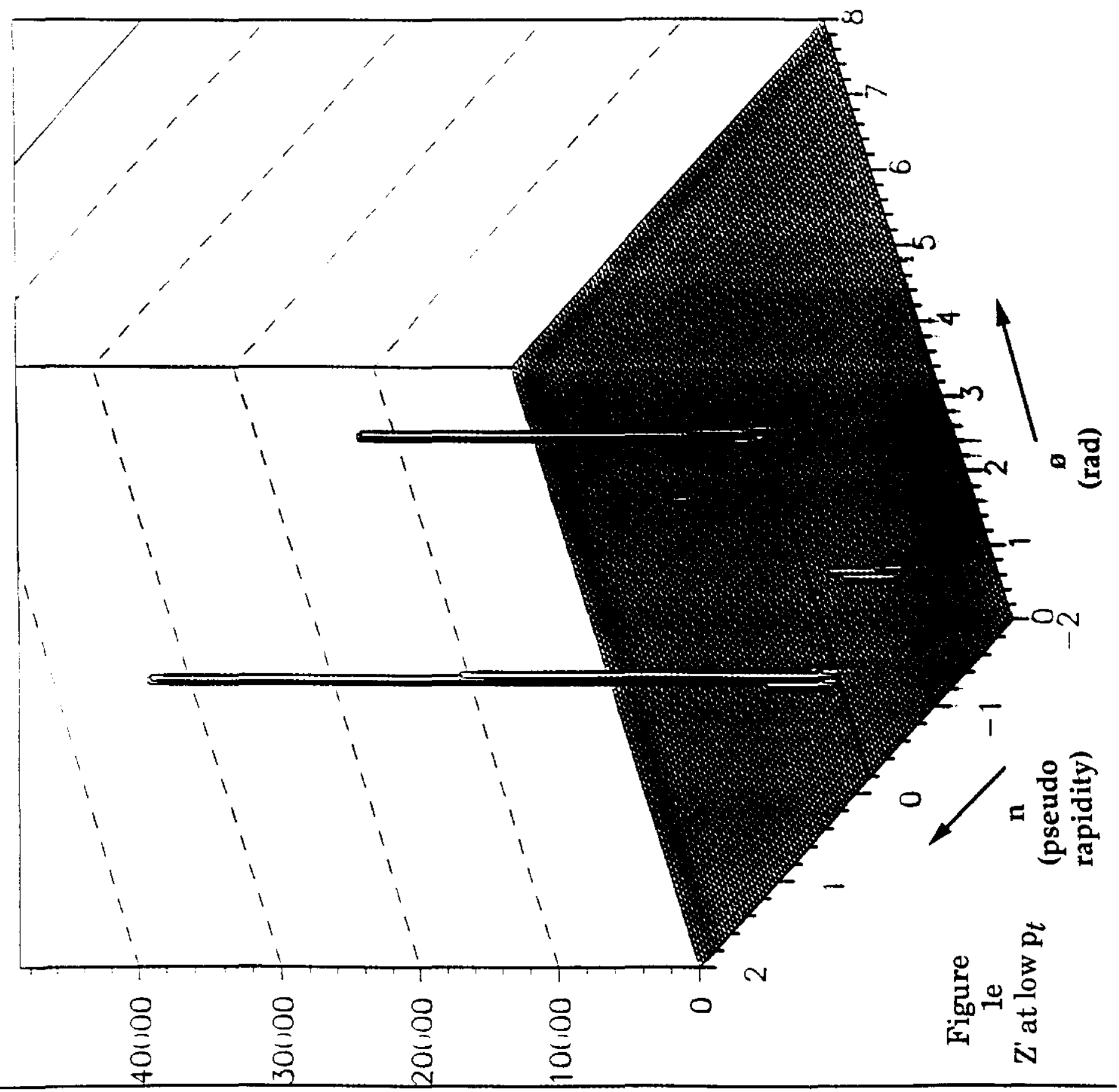




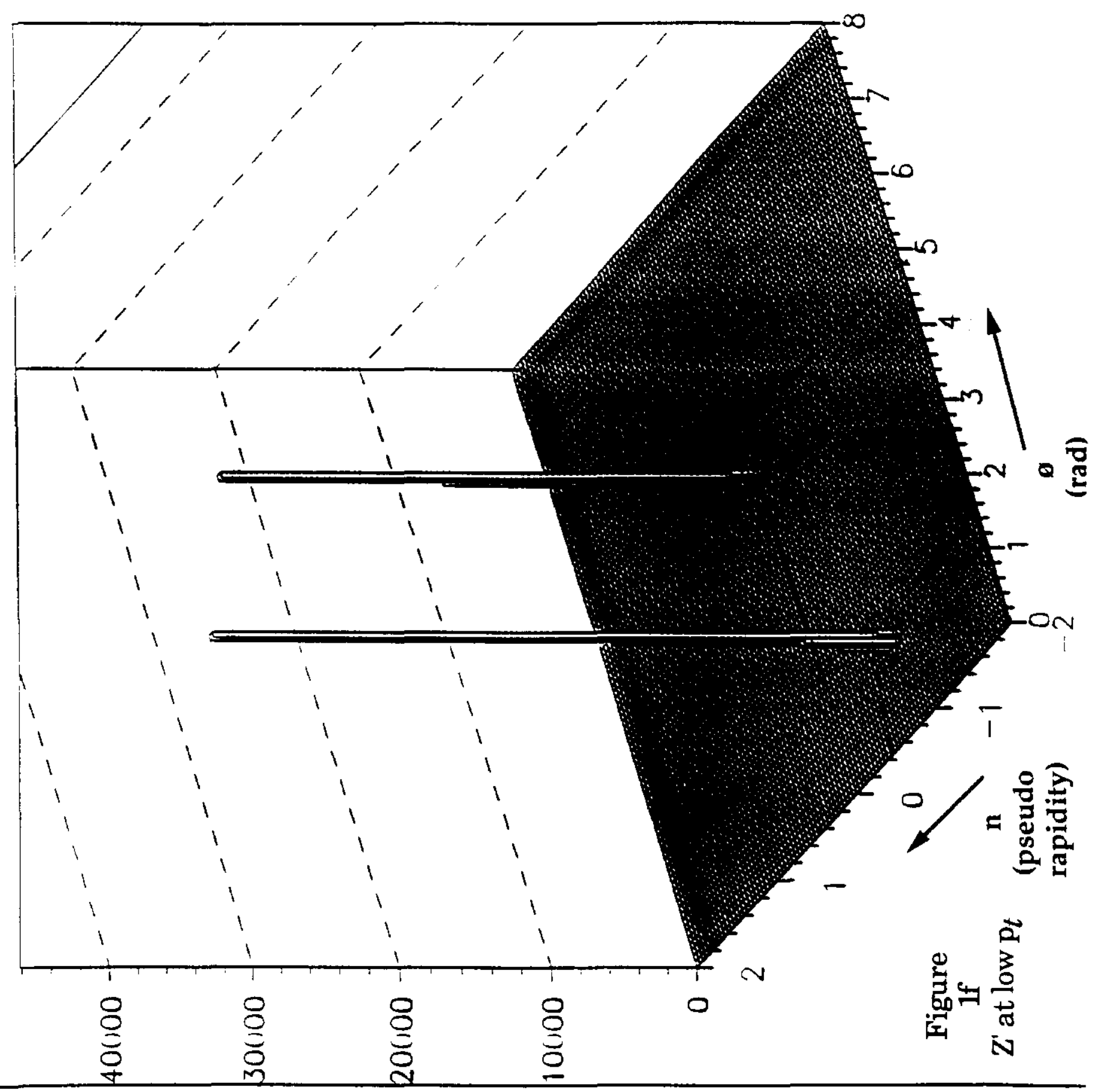




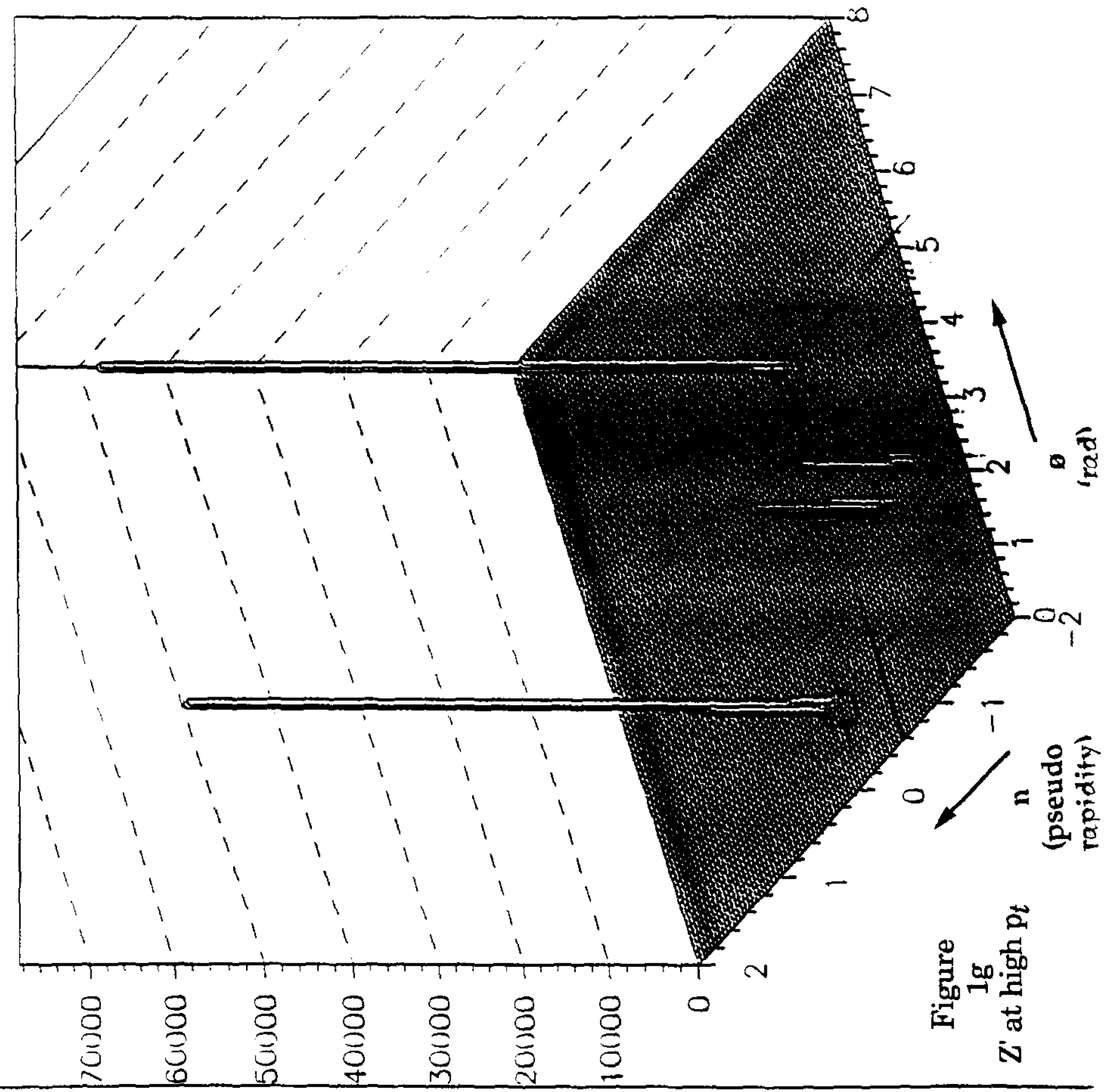




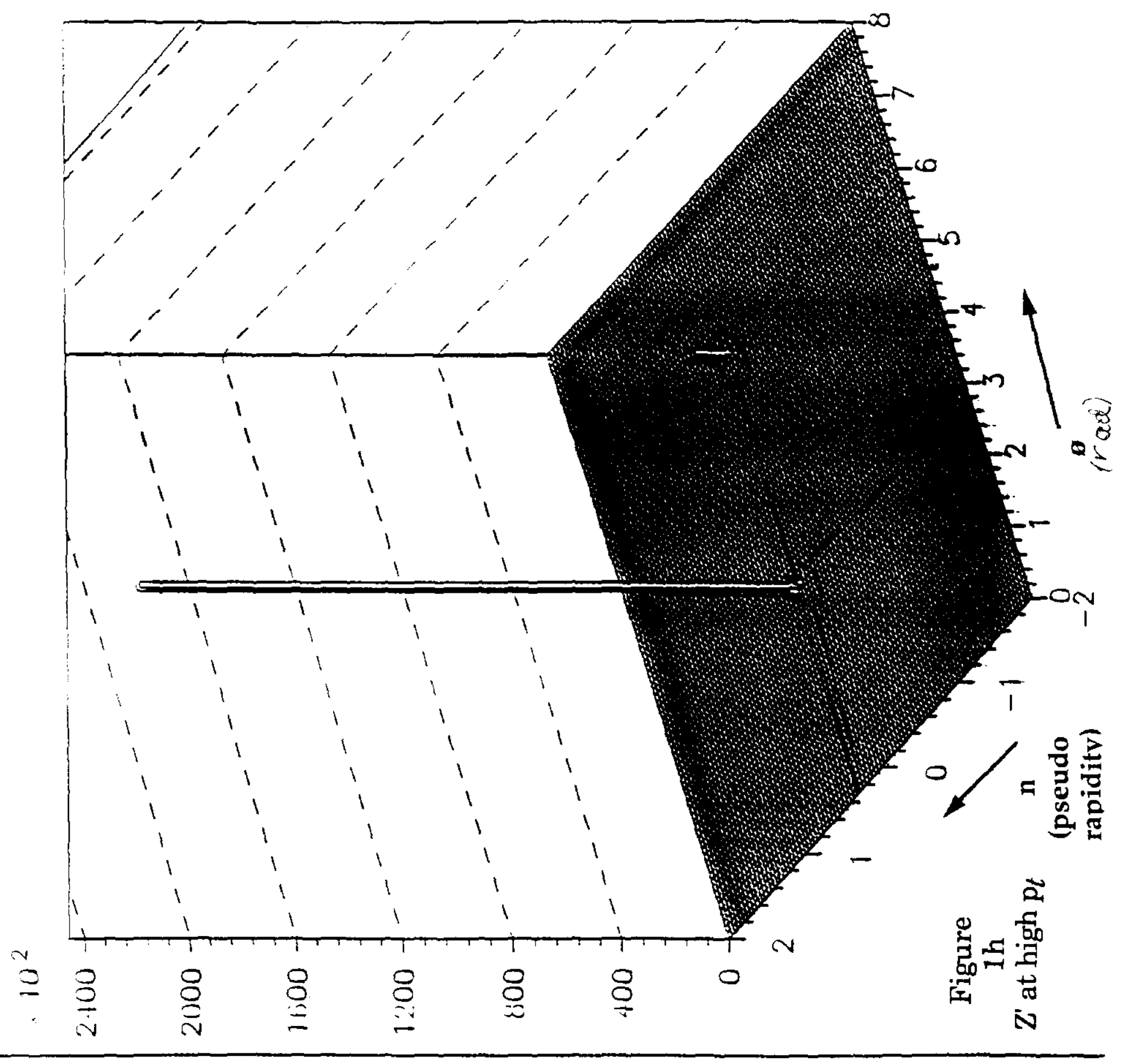




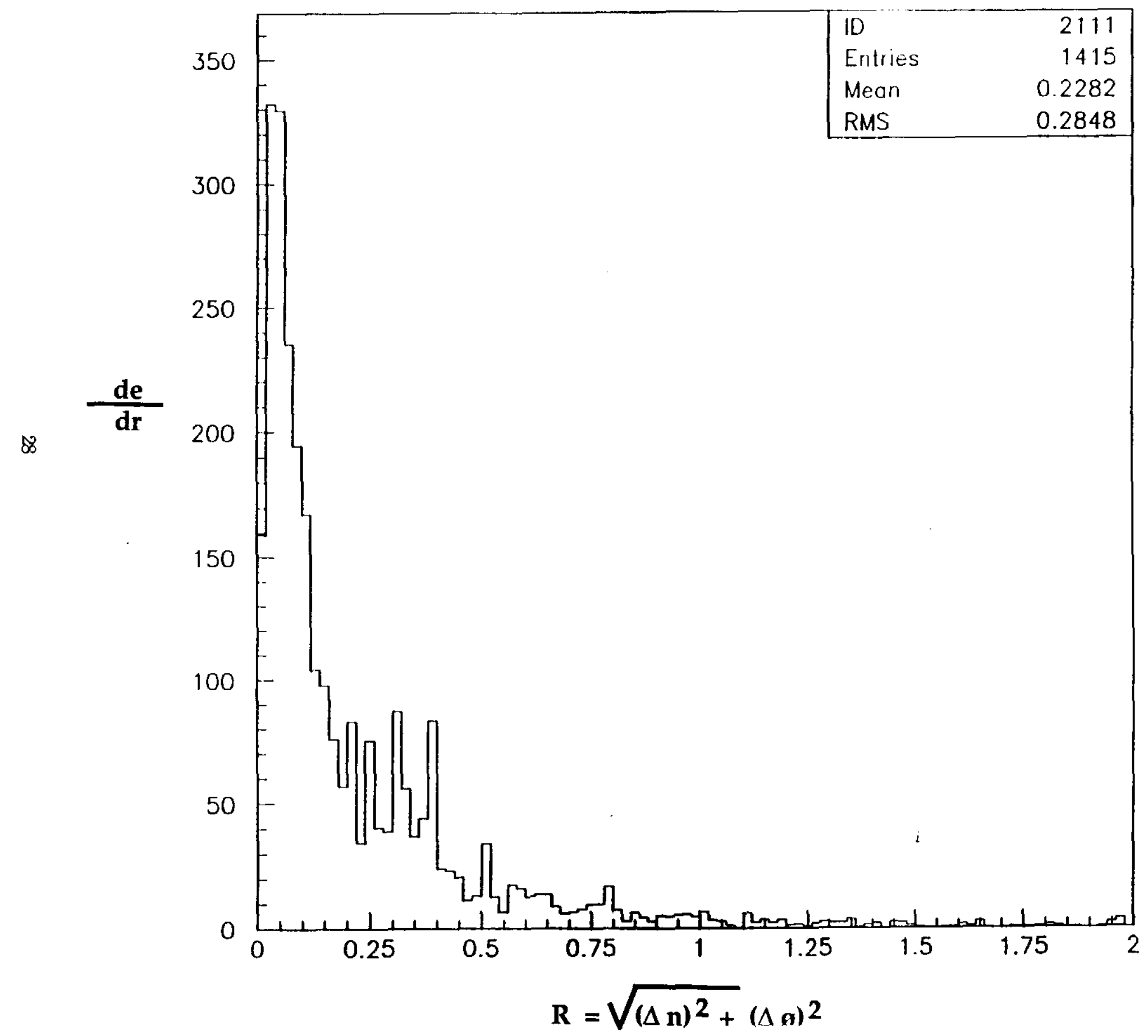

Figure 


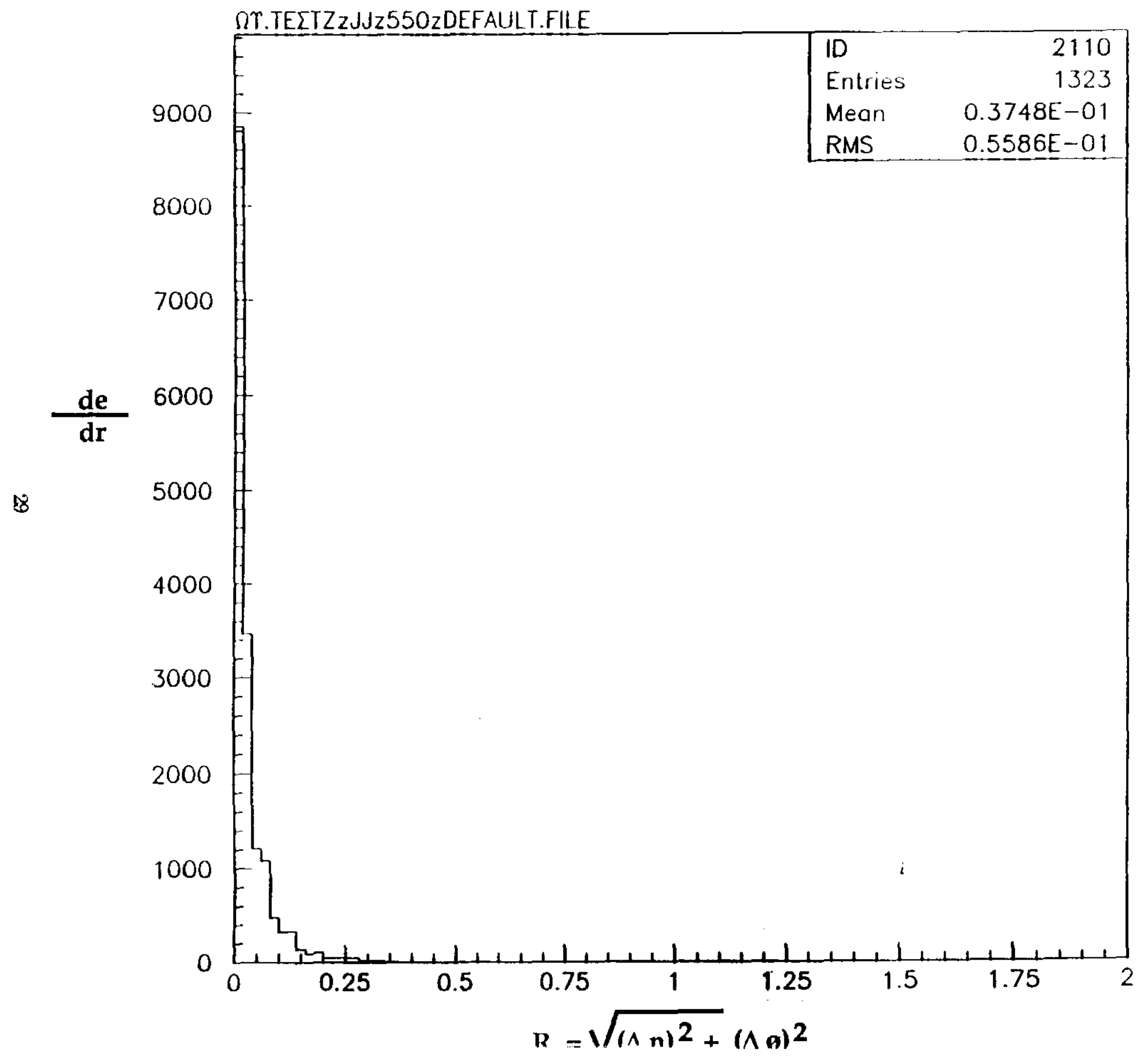

Figure
$2 \mathrm{~b}$

$\mathrm{Z}$ at high $\mathrm{p}_{t}$ 


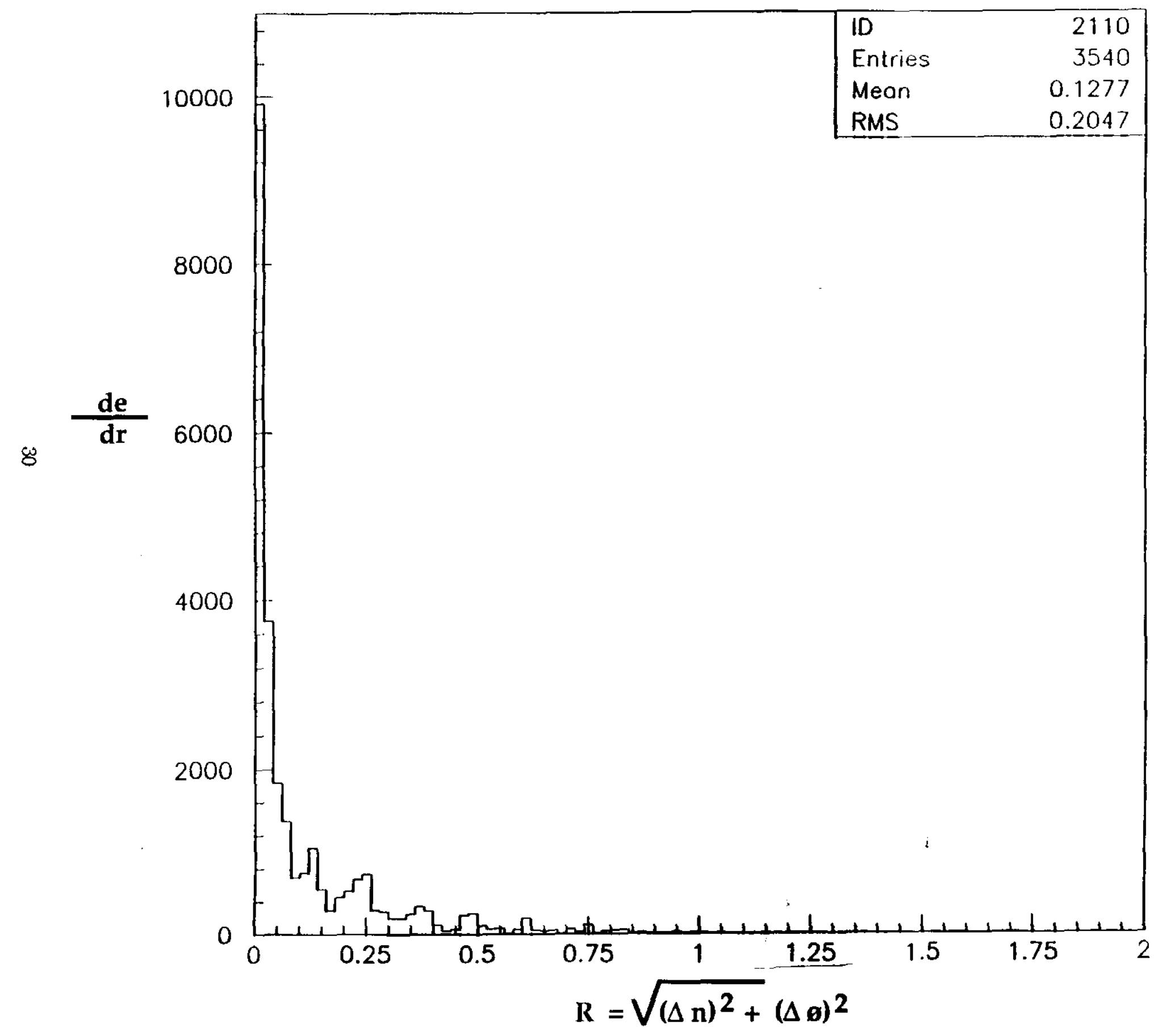

Figure 


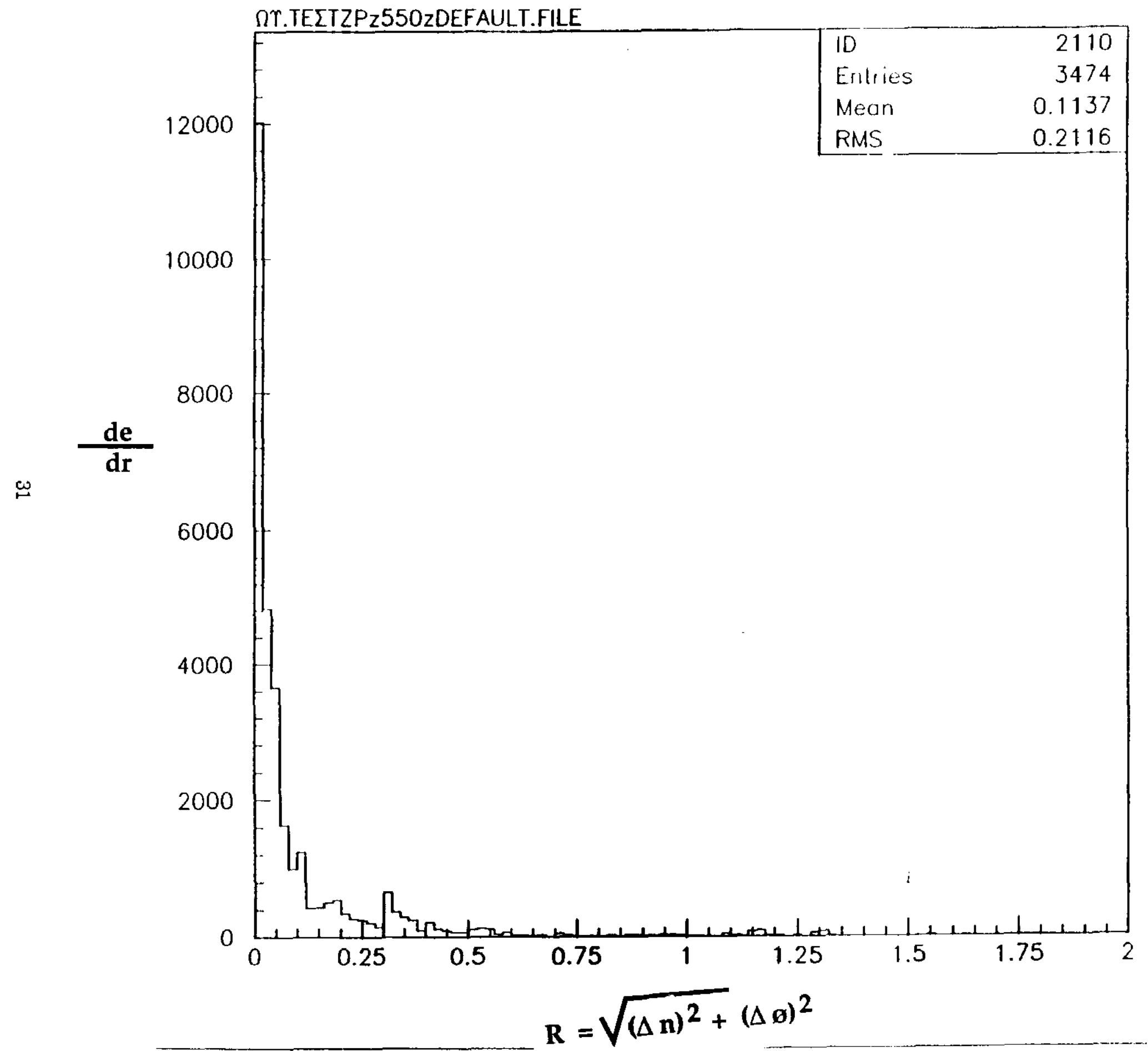

Figure 


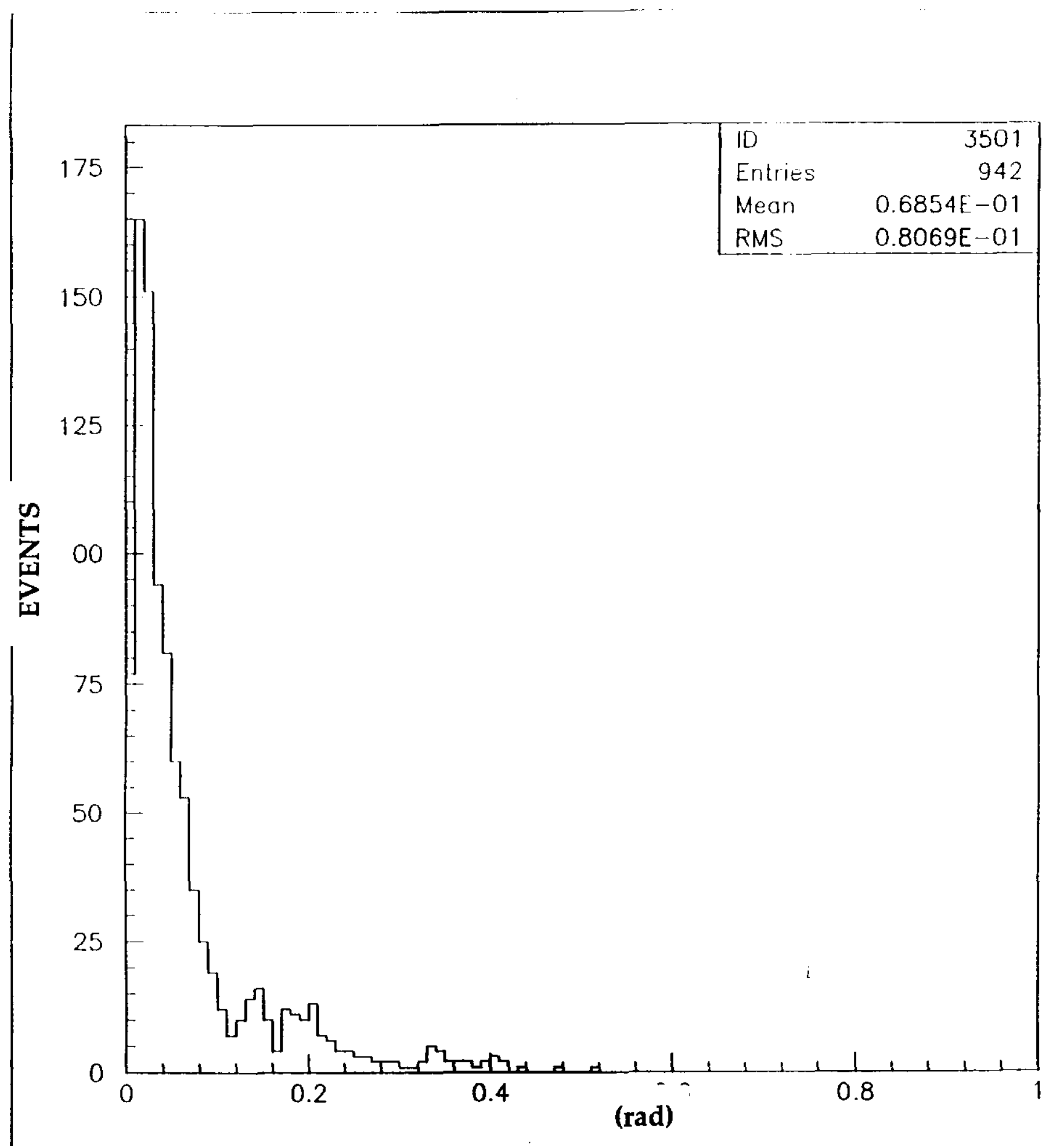

Figure 


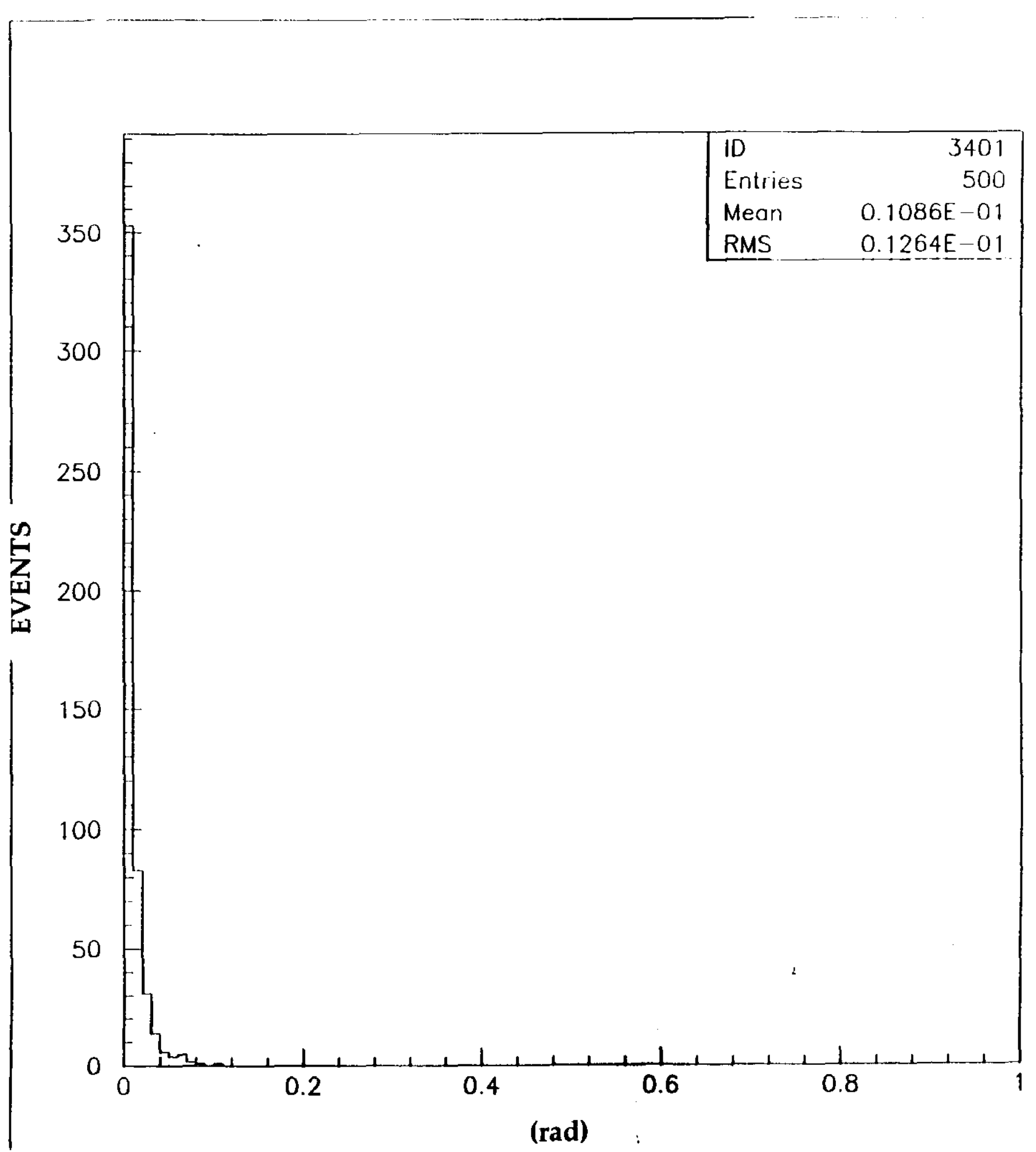

Figure

$3 b$

$\mathrm{Z}$ at high $\mathrm{p}_{t}$ 


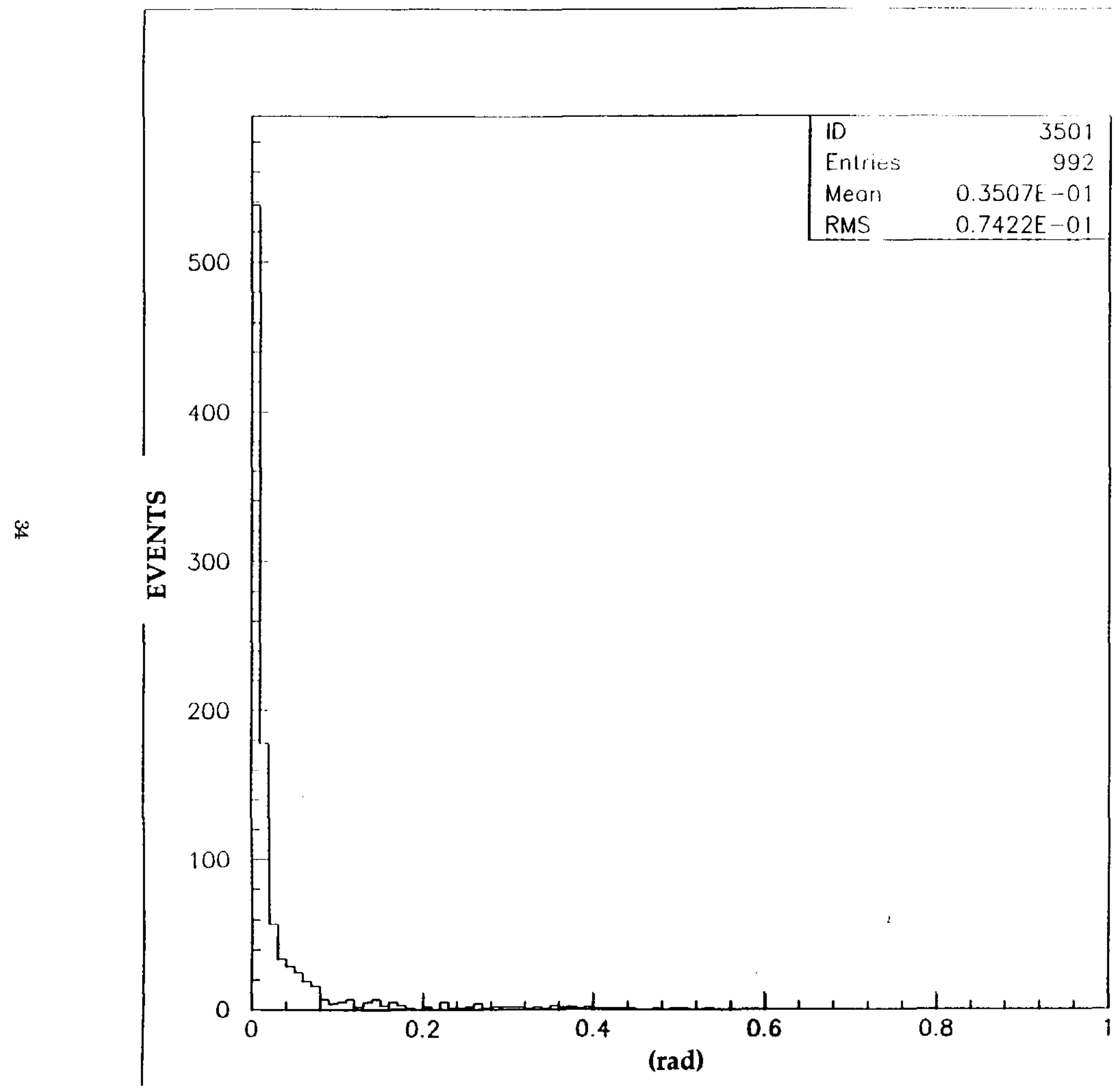

Figure $3 c$ $\mathrm{Z}$ at low $\mathrm{p}_{t}$ 


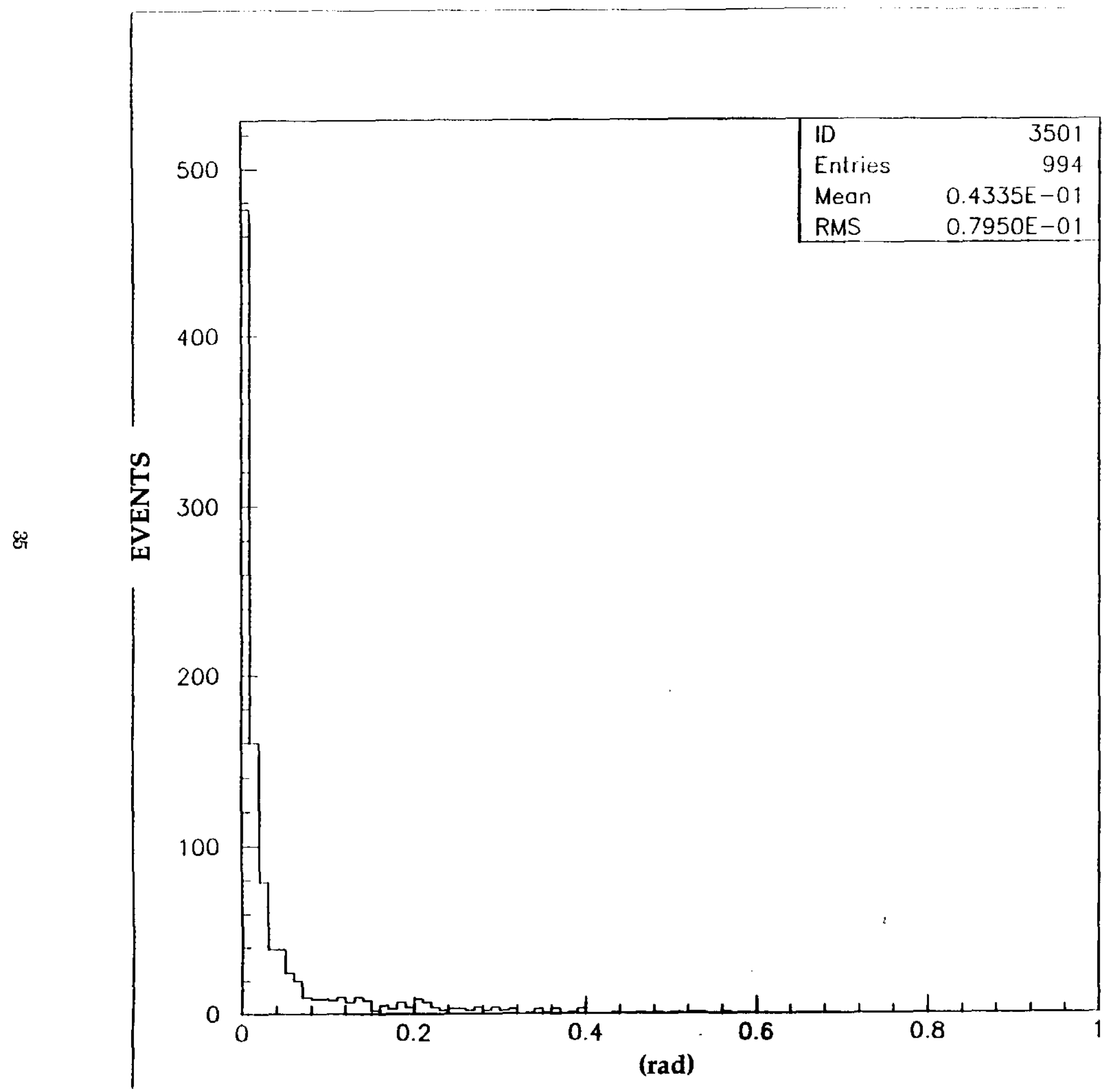

Figure $3 d$

$\mathrm{Z}$ at high $\mathrm{p}_{t}$ 


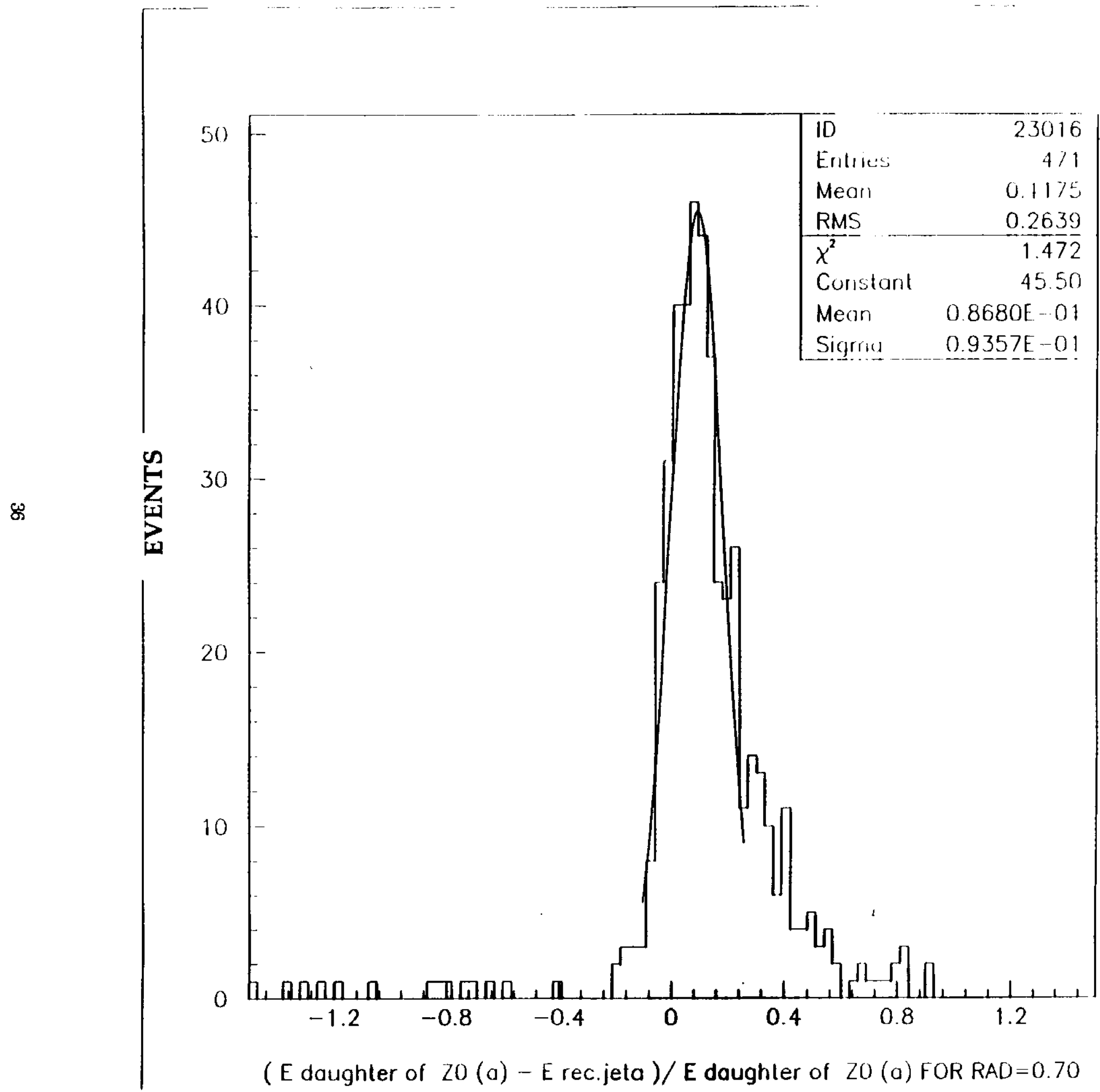

Figure 


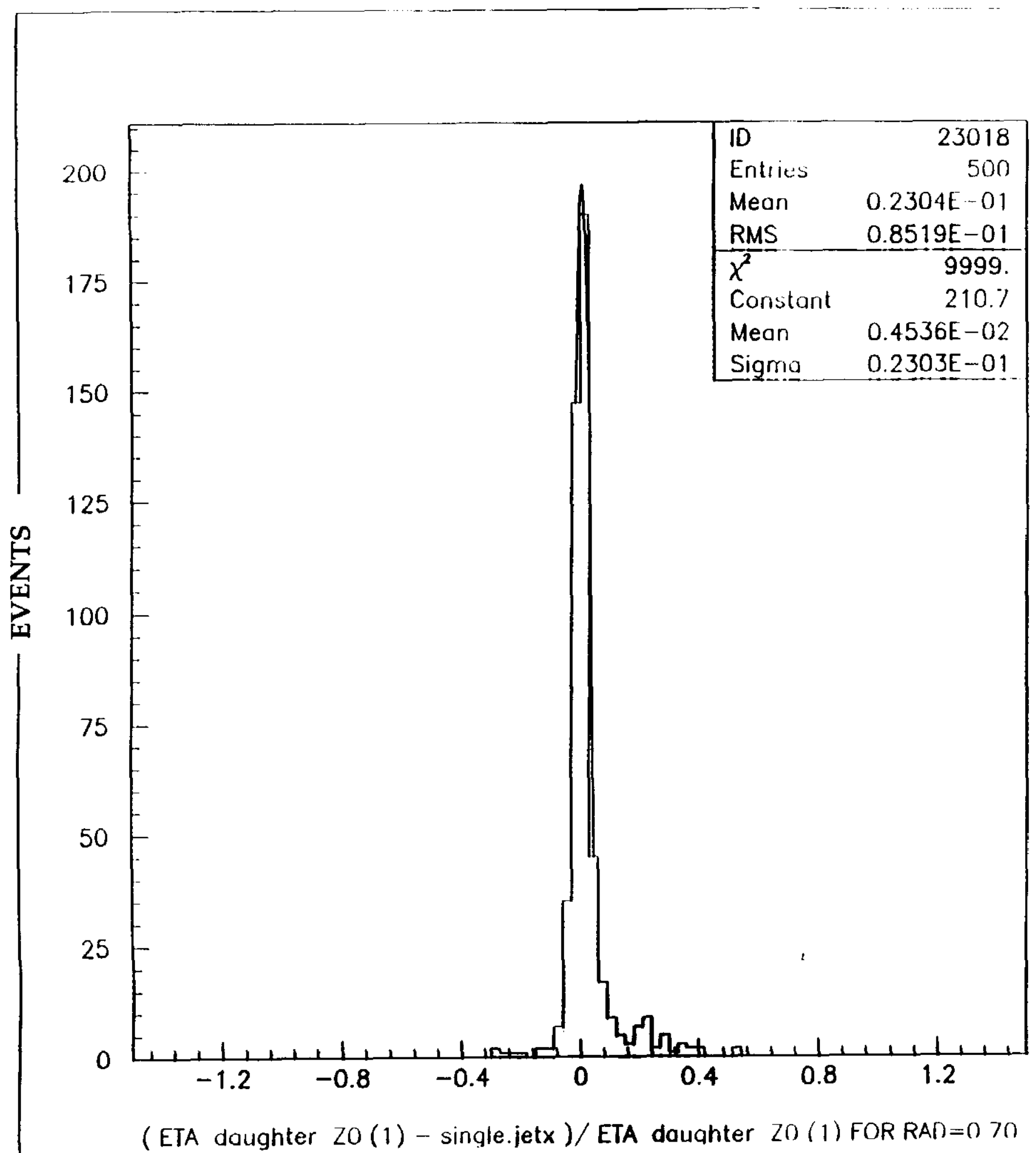

Figure $4 \mathrm{~b}$

$\mathrm{Z}$ at high $\mathrm{p}_{t}$ 


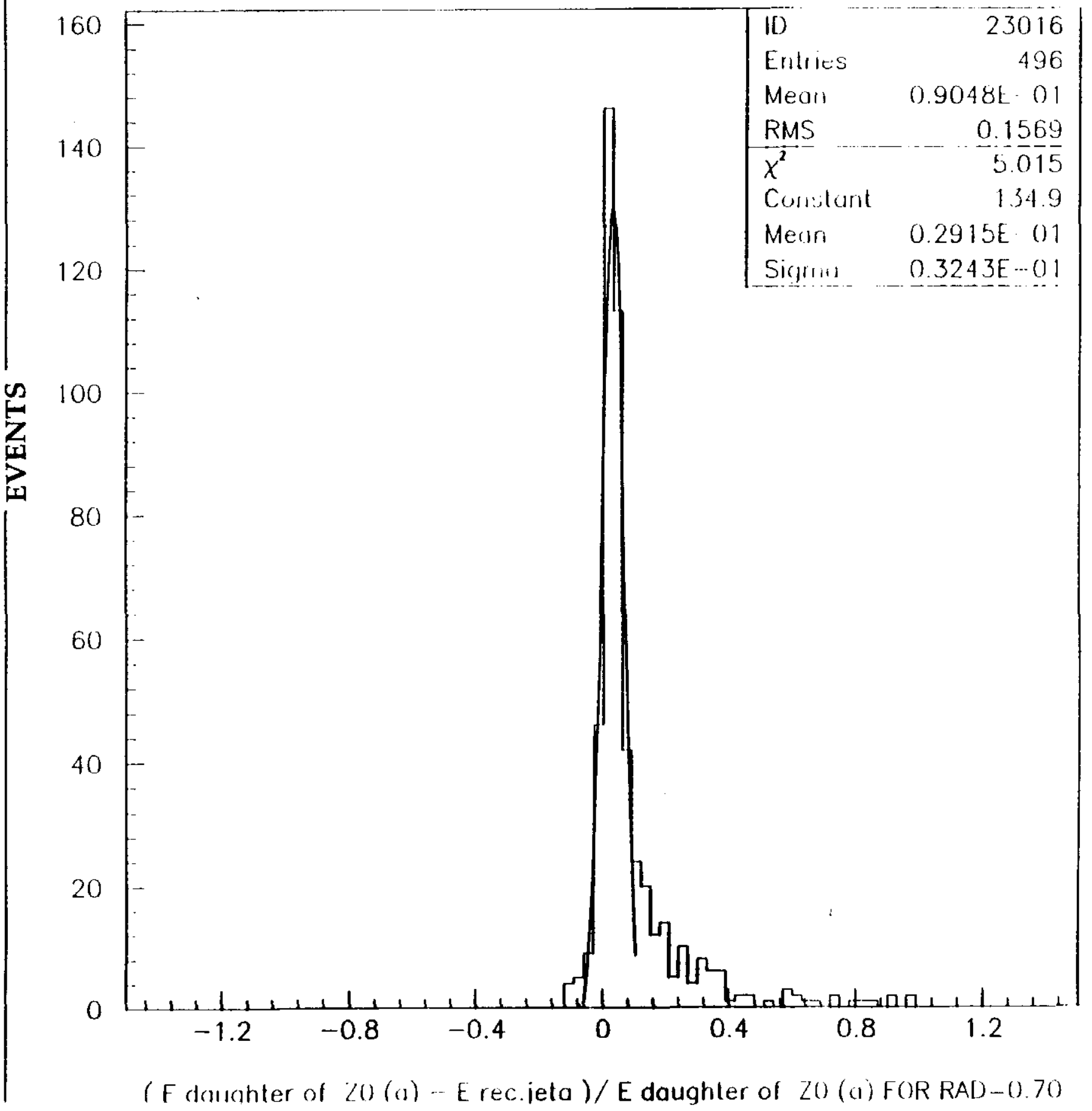

Figure 


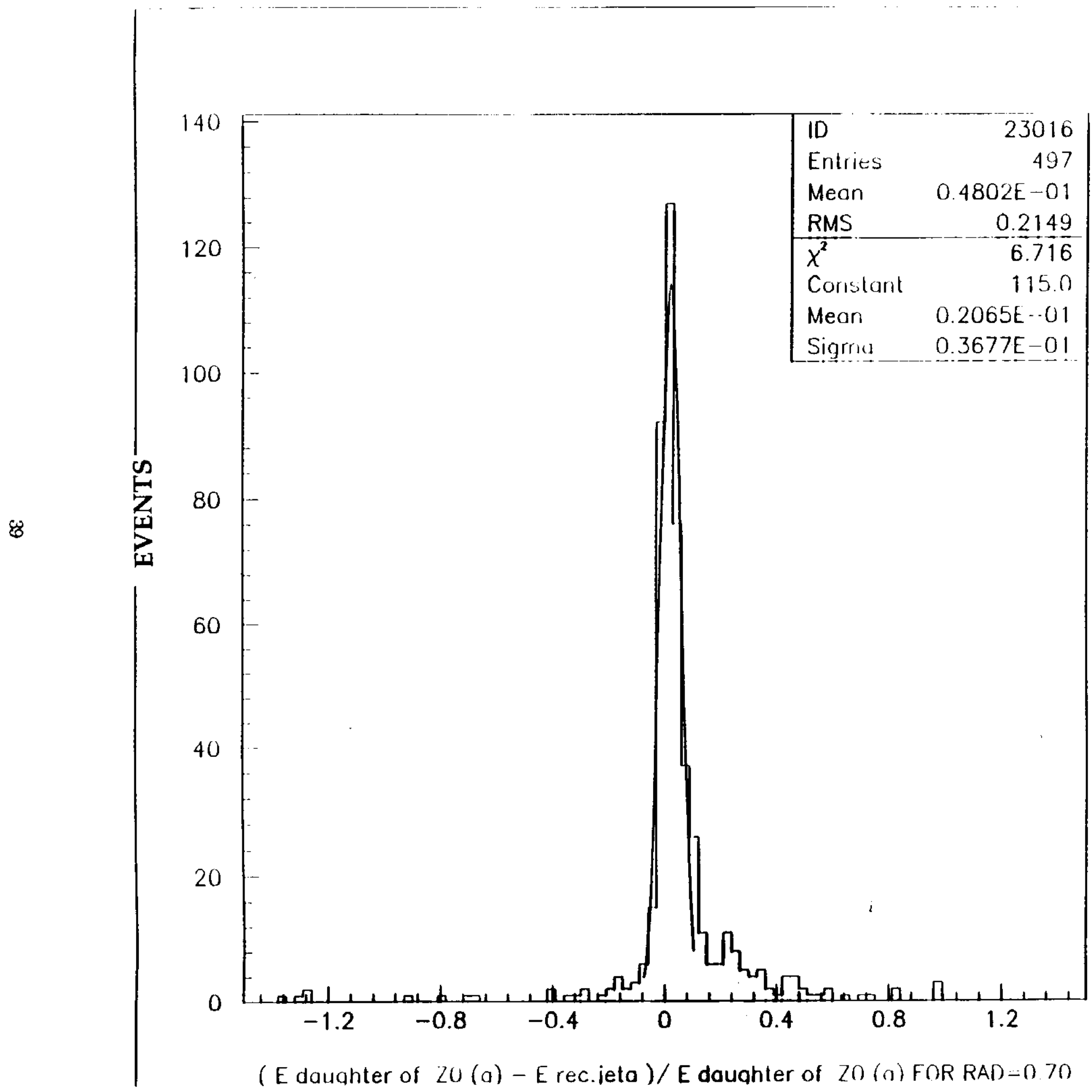

Figure $4 \mathrm{~d}$ $\mathrm{Z}^{\prime}$ at high $\mathrm{p}_{t}$ 


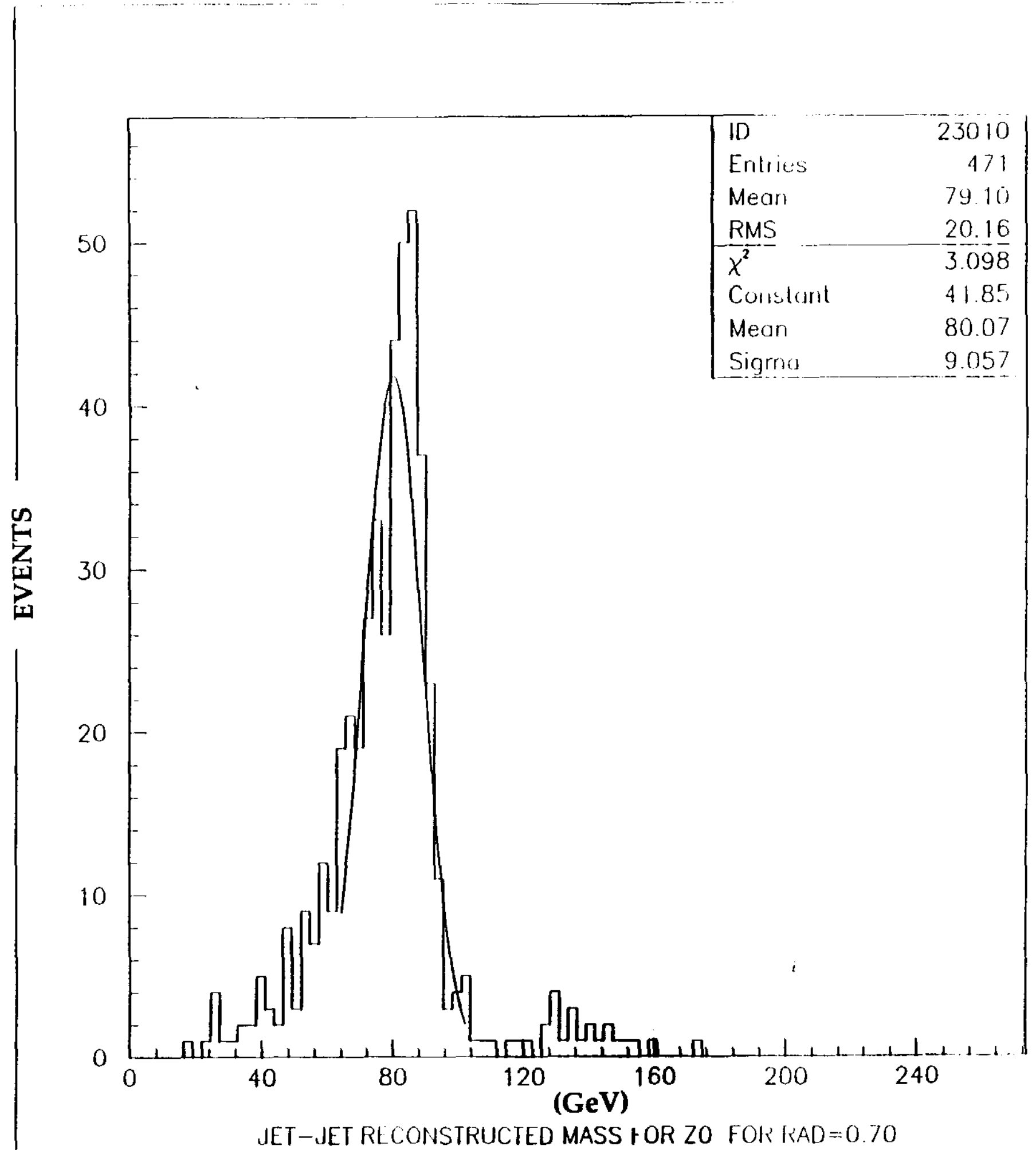

Figure 


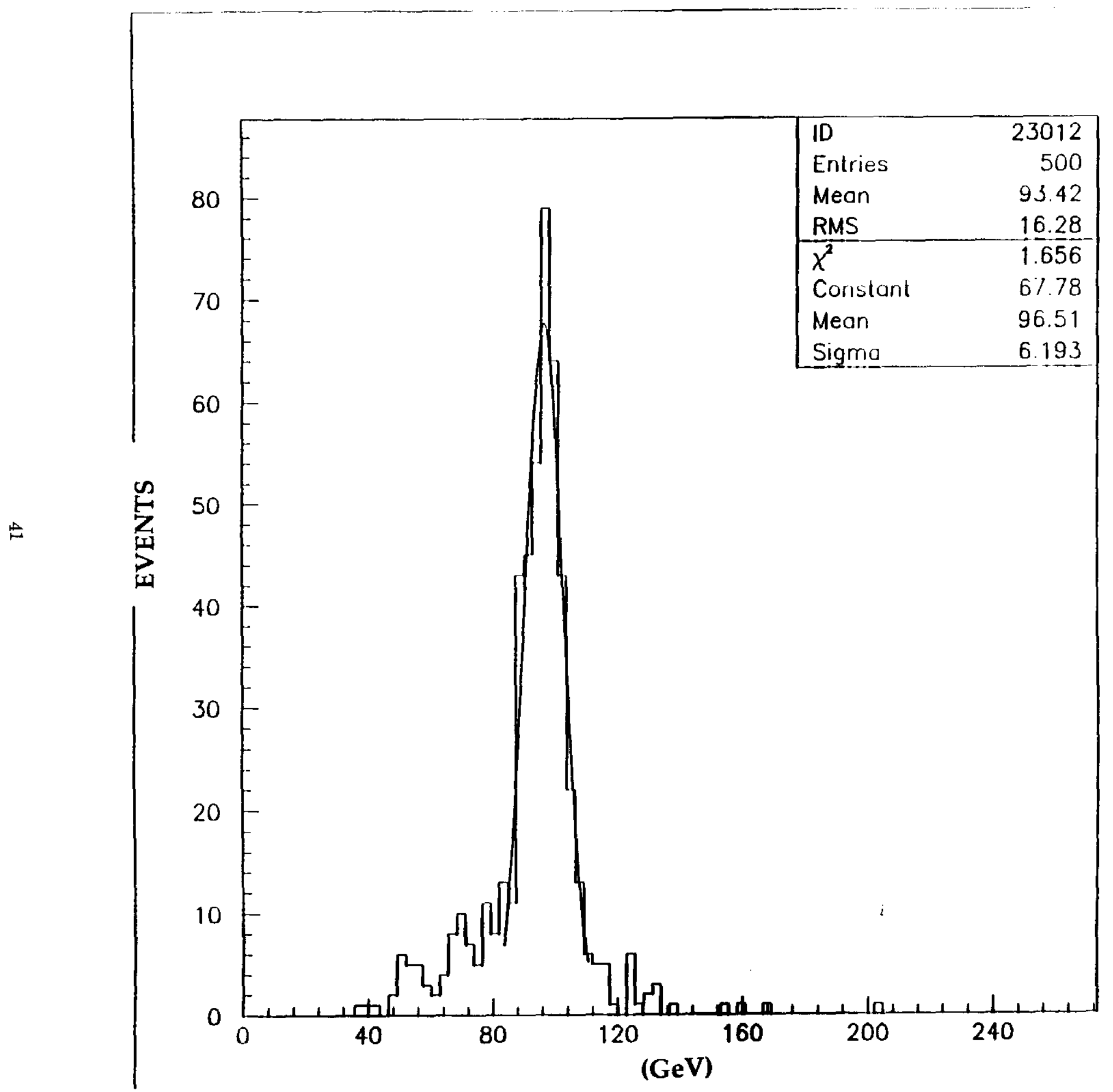

Figure $5 b$ $\mathrm{Z}$ at high $\mathrm{p}_{t}$ 


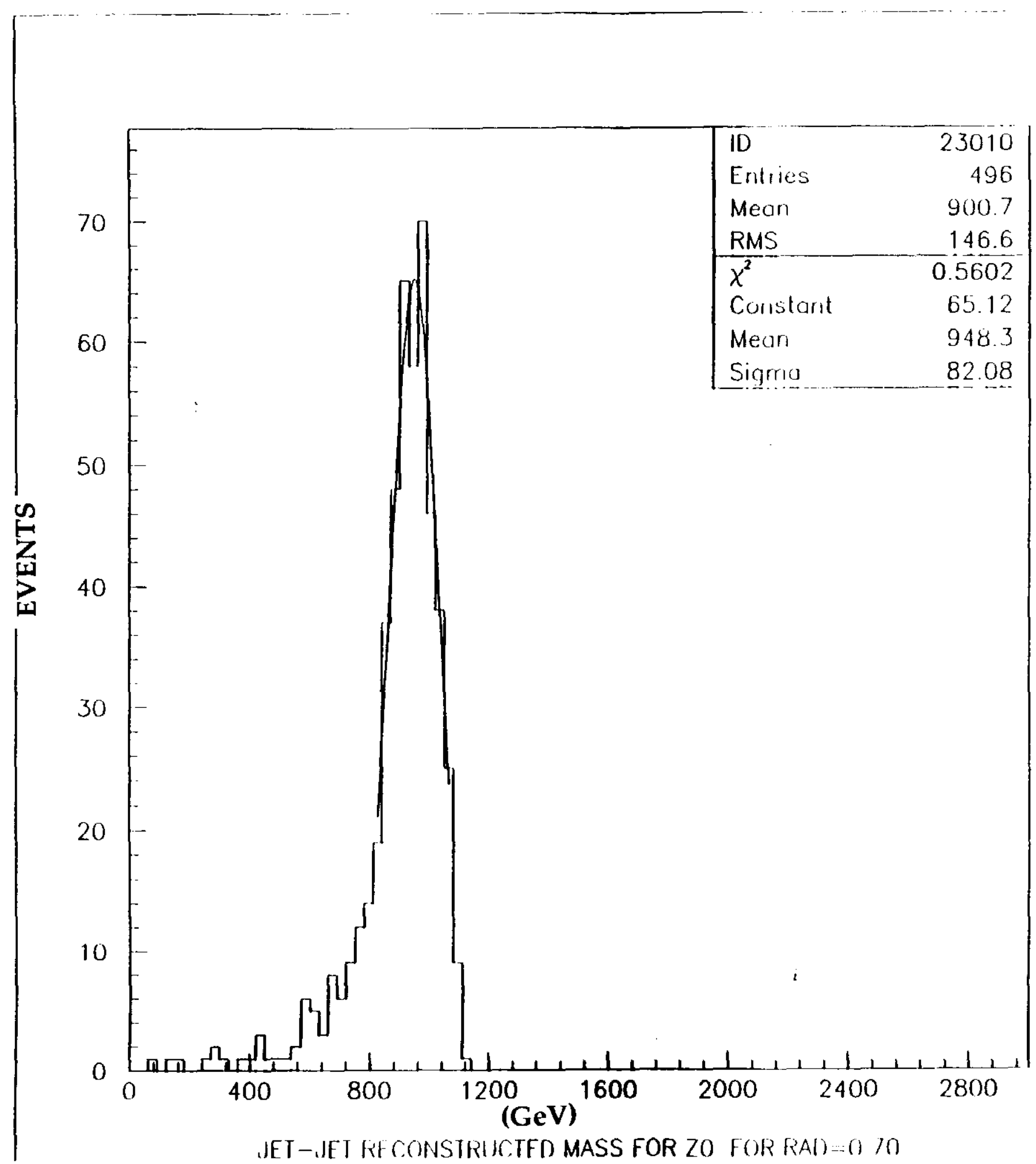

Figure 


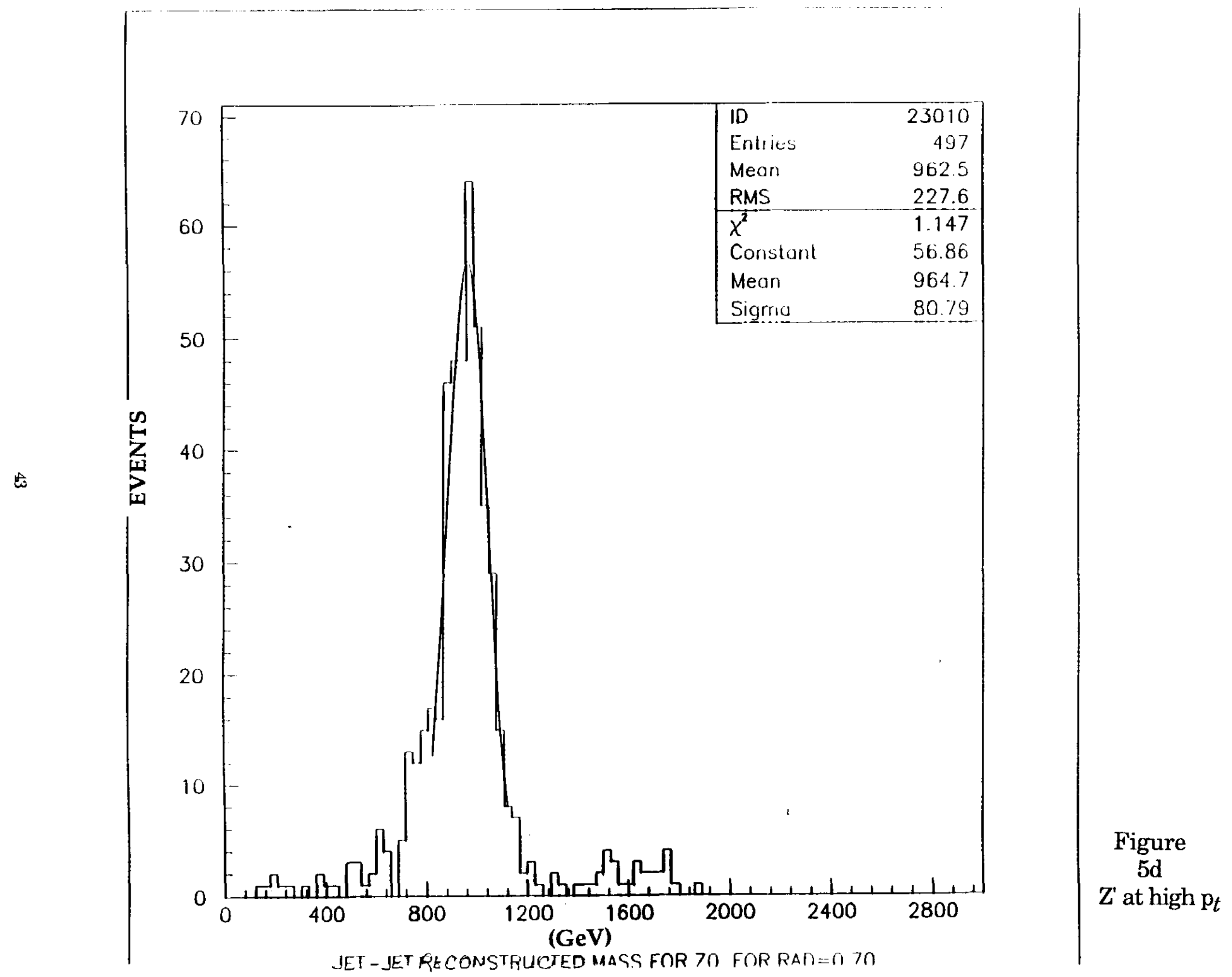




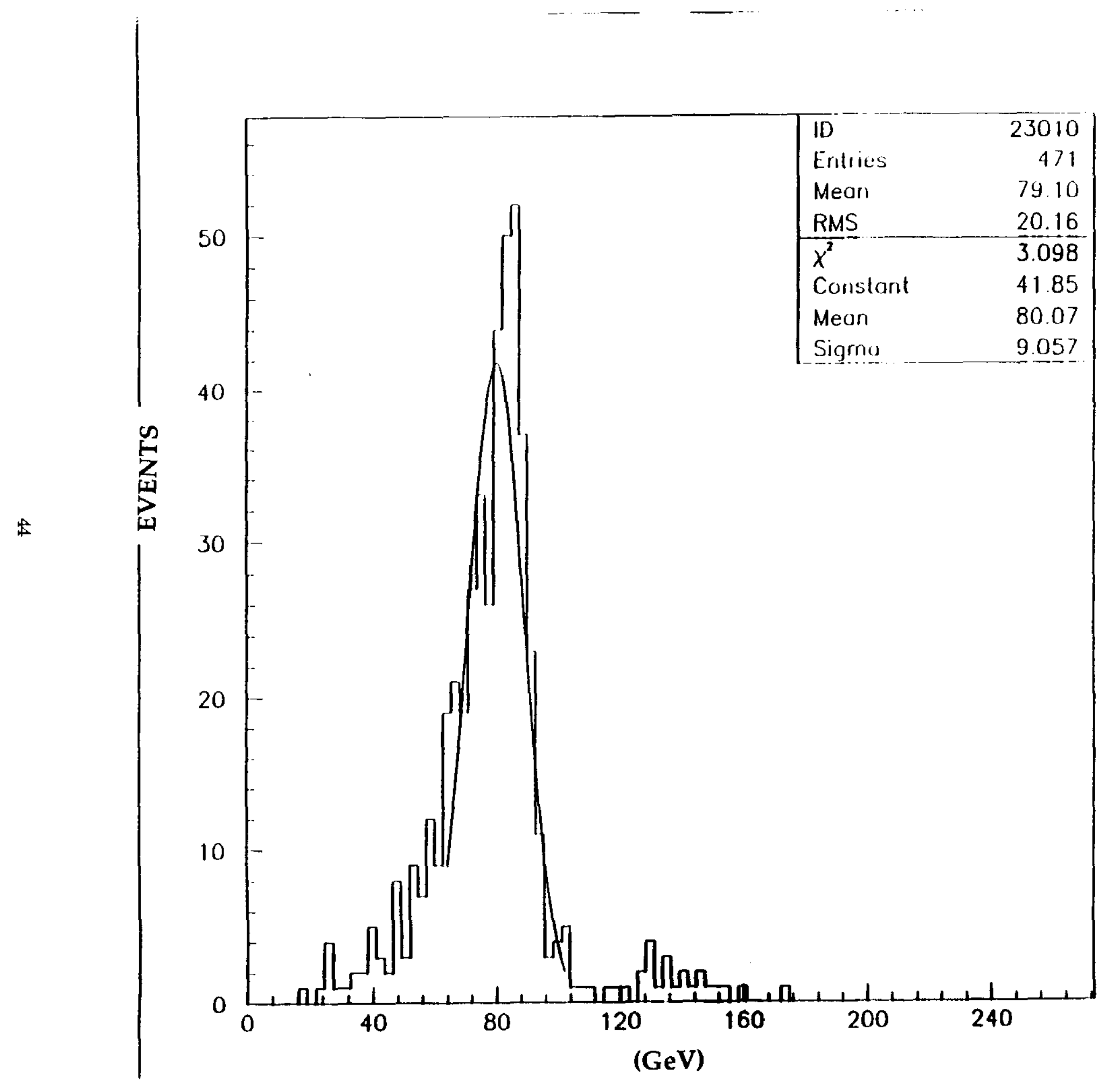

Figure $6 a$ $\mathrm{Z}$ at low $\mathrm{p}_{t}$ 


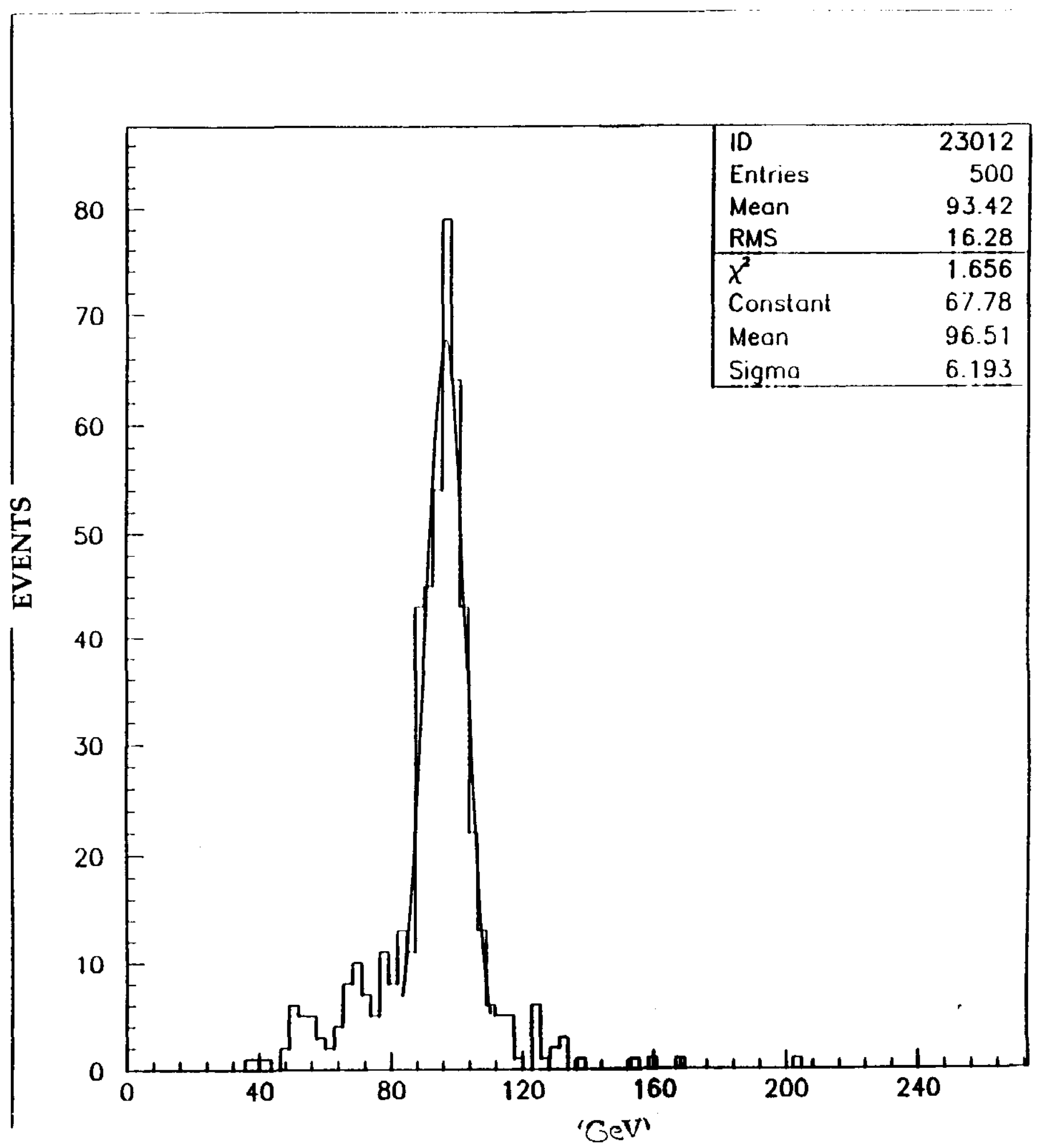

Figure 
客

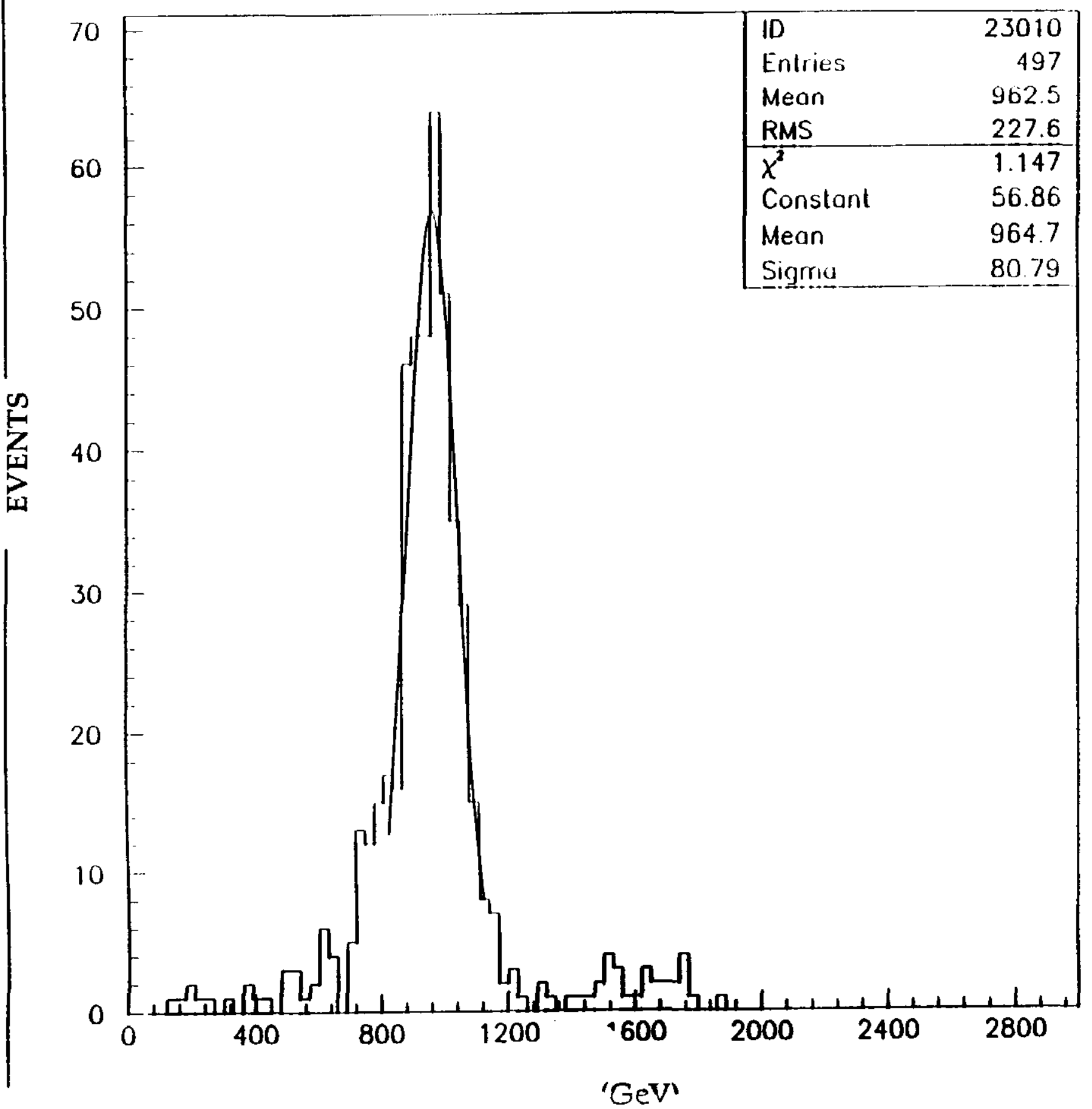

Figure $6 \mathrm{~d}$

$\mathrm{Z}$ at high $\mathrm{p}_{t}$ 


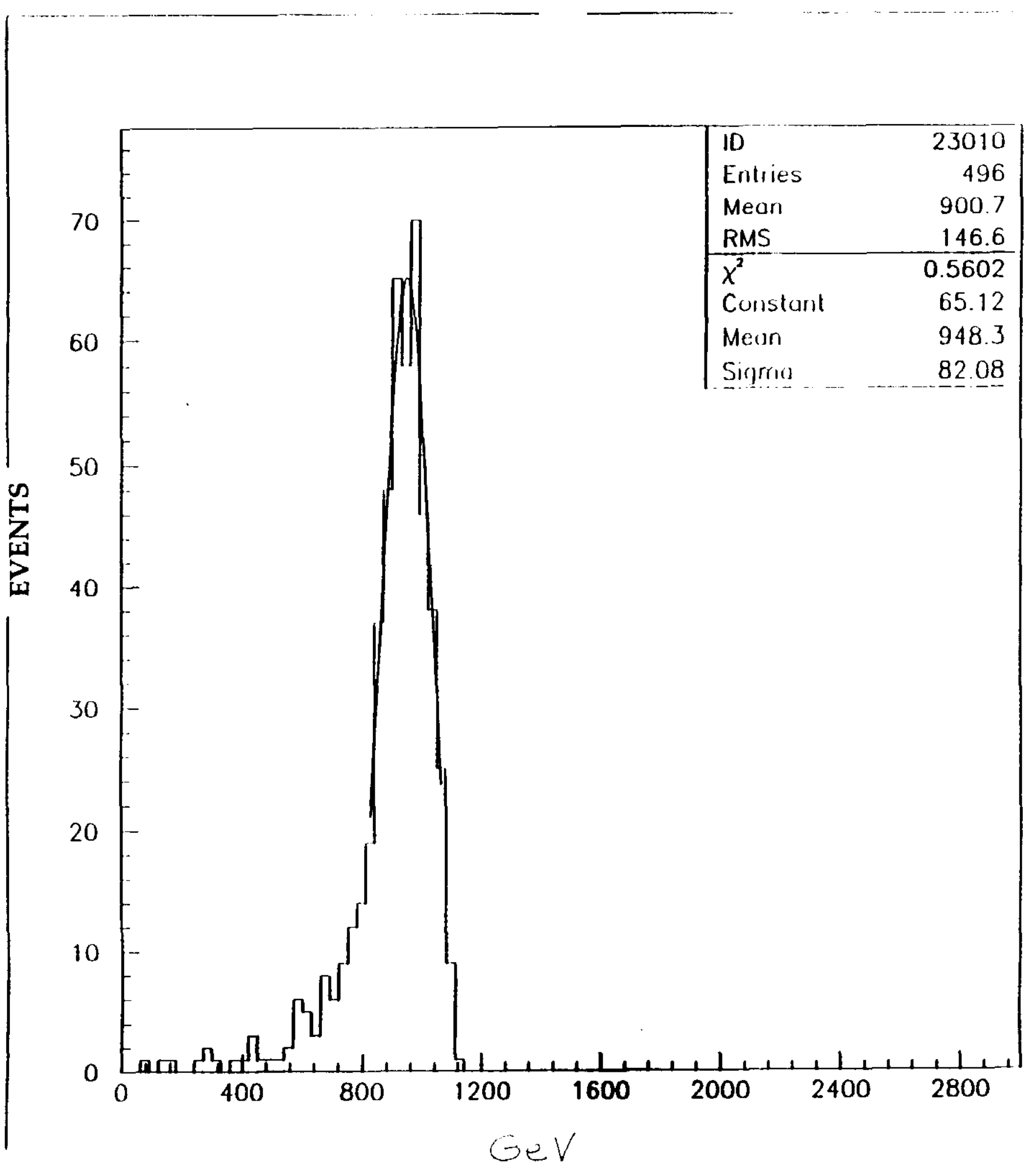

Figure 